\title{
Femtosecond Transition-state Dynamics
}

\author{
Ahmed H. Zewail \\ Arthur Amos Noyes Laboratory of Chemical Physics, California Institute of Technology, \\ Pasadena, California 91125, USA
}

\begin{abstract}
This article presents the progress made in probing femtosecond transitionstate dynamics of elementary reactions. Experiments demonstrating the dynamics in systems characterized by a transition region and by a saddlepoint transition state are reported, and comparison with theory is made.
\end{abstract}

\section{Introduction}

Since the early days of the transition-state theory of Eyring, Evans and Polanyi, ${ }^{1}$ the fleeting nature of the state as the reaction proceeds from reagents to products has been known. The lifetime of the state is typically $<10^{-12} \mathrm{~s}$, and hence to observe these transition states in real time the temporal resolution must be on the femtosecond scale. Progress has been made in probing transition states of elementary reactions and in studying the dynamics in real time. ${ }^{2}$

The objective of this account is not to review all the work done in this area (for a review, see ref. 2). Instead, I wish to discuss recent developments with particular focus on two- and one-dimensional reactions. The dimensionality (D) of the potential-energy surface (PES) determines the nature of the transition state, TS. In 1-D systems there is only one vibrational coordinate, and the motion can be mapped out as atoms recoil along this coordinate in the transition region. In 2-D (or more) systems, there may be a saddle point, and the dynamics would involve both the translational motion along the antisymmetric coordinate and vibrational motion (dynamical resonance) along the symmetric coordinate. In this case the saddle point defines clearly a TS, and the system spans both bound and repulsive features of the PES. Experiments demonstrating the dynamics in such systems are reported, and comparison with theory is made.

\section{A Prototype Case: the Transition Region of Alkali Halide Reactions}

We consider first the simplest case of two atoms with a single internuclear coordinate in the half-collision dissociation of a diatomic. This example we use to demonstrate the concepts involved and to report on the recent progress made on these alkali halide (MX) reactions:

$$
\mathrm{MX}^{*} \rightarrow[\mathrm{M} \cdots \mathrm{X}]^{\ddagger *} \rightarrow \mathrm{M}+\mathrm{X}
$$

As discussed by Berry, ${ }^{3}$ the PES for this class of reactions is well described by the covalent $(\mathrm{M}+\mathrm{X})$ and ionic $\left(\mathrm{M}^{+}+\mathrm{X}^{-}\right)$curves, which cross at a given internuclear distance, $R_{\mathrm{x}}$ (Fig. 1). Actually there are more curves, but they are not relevant here. When an alkali atom (e.g. $\mathrm{Na}$ ) is brought closer to a halogen atom (e.g. I), according to the molecular-states picture [Hund's case (a); $\Lambda=0(\Sigma)$ and $\Lambda=1(\Pi)$ etc.], $\Pi$ and $\Sigma$ singlet and triplet states are formed. However because of spin-orbit coupling, Hund's case (c) is invoked, and the states are actually indexed according to the projection of the total angular momentum along the bond axis giving rise to $\Omega=0^{+}$and 1 states. In a correlation scheme, the $0^{+}$state correlates with the $\mathrm{B}^{1} \Sigma^{+}$and the 1 state correlates with the $A^{1} \Pi$ (and triplet) state. The ground state is of type $\Sigma$ (or 0 ), and therefore 


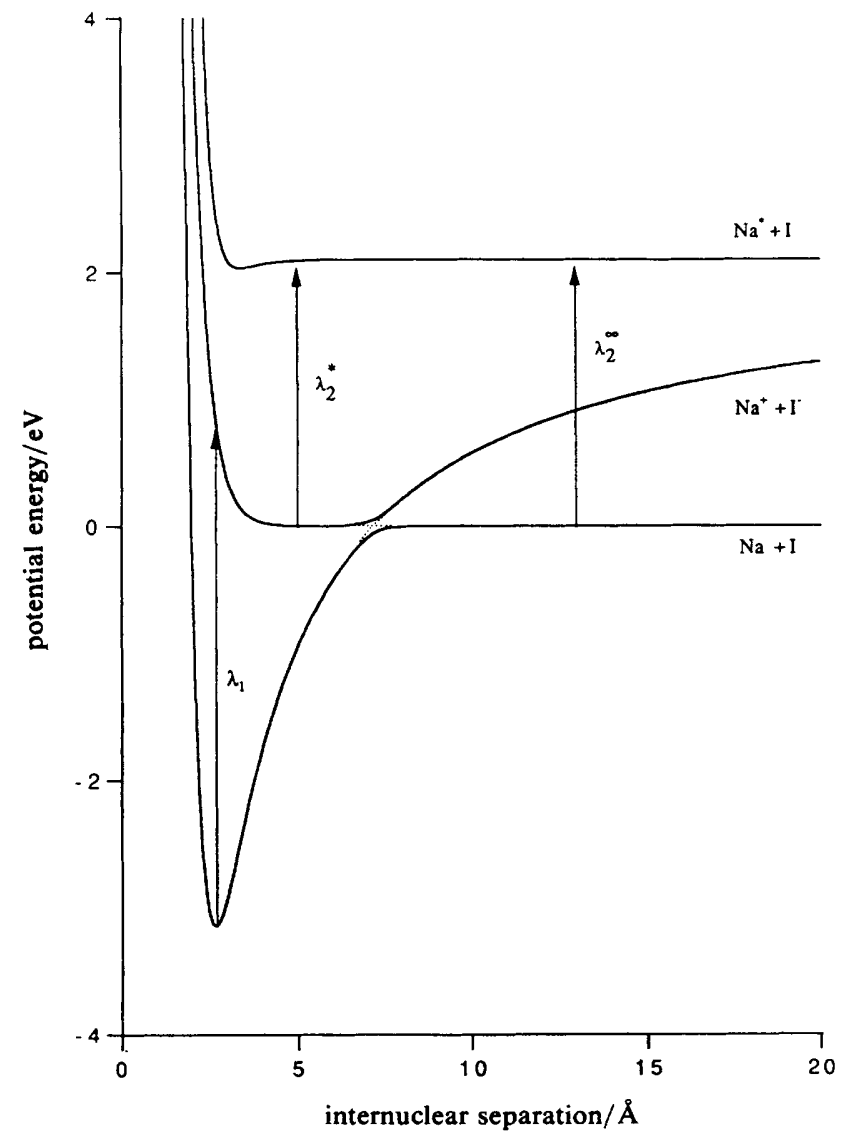

Fig. 1 The covalent $(M+X)$ and ionic $\left(M^{+}+X^{-}\right)$potential energy curves for the reaction of alkali halides

the covalent $0^{+}$and the ground state interact at the crossing point $R_{\mathrm{x}}$ to form the avoided crossing region between the covalent and ionic curves (Fig. 1). The covalent curve is essentially non-bonding (except for van der Waals attraction) until it reaches the repulsive branch.

This reaction of alkali halides represents an ideal test case for using femtosecond transition-state spectroscopy (FTS) to probe the nuclear motion as the system proceeds from $\mathrm{MX}^{*}$ to $[\mathrm{M} \cdots \mathrm{X}] \ddagger^{*}$ and then finally to $\mathrm{M}+\mathrm{X}$. Over the past years we have studied these systems with different time and spatial resolutions in order to observe the nuclear motion in real time. In this section we discuss the femtosecond dynamics, particularly for the $\mathrm{NaI}$ system, and the new findings.

\subsection{The Activated Complex: Motion between the Covalent and Ionic Potentials}

As the wave packet, prepared at time zero, moves from the covalent to the ionic curve, the bond changes its characteristic from being purely covalent to a mixture of covalent and ionic. As discussed by Pauling, ${ }^{4}$ the crossing (or closeness) of the covalent and ionic potentials is a general phenomenon which describes the nature of the chemical bond. The dynamics of the bond is that of the activated complex, as termed by Atkins, ${ }^{5}$ in the transition region and its decay toward final products. Classically, the probability 

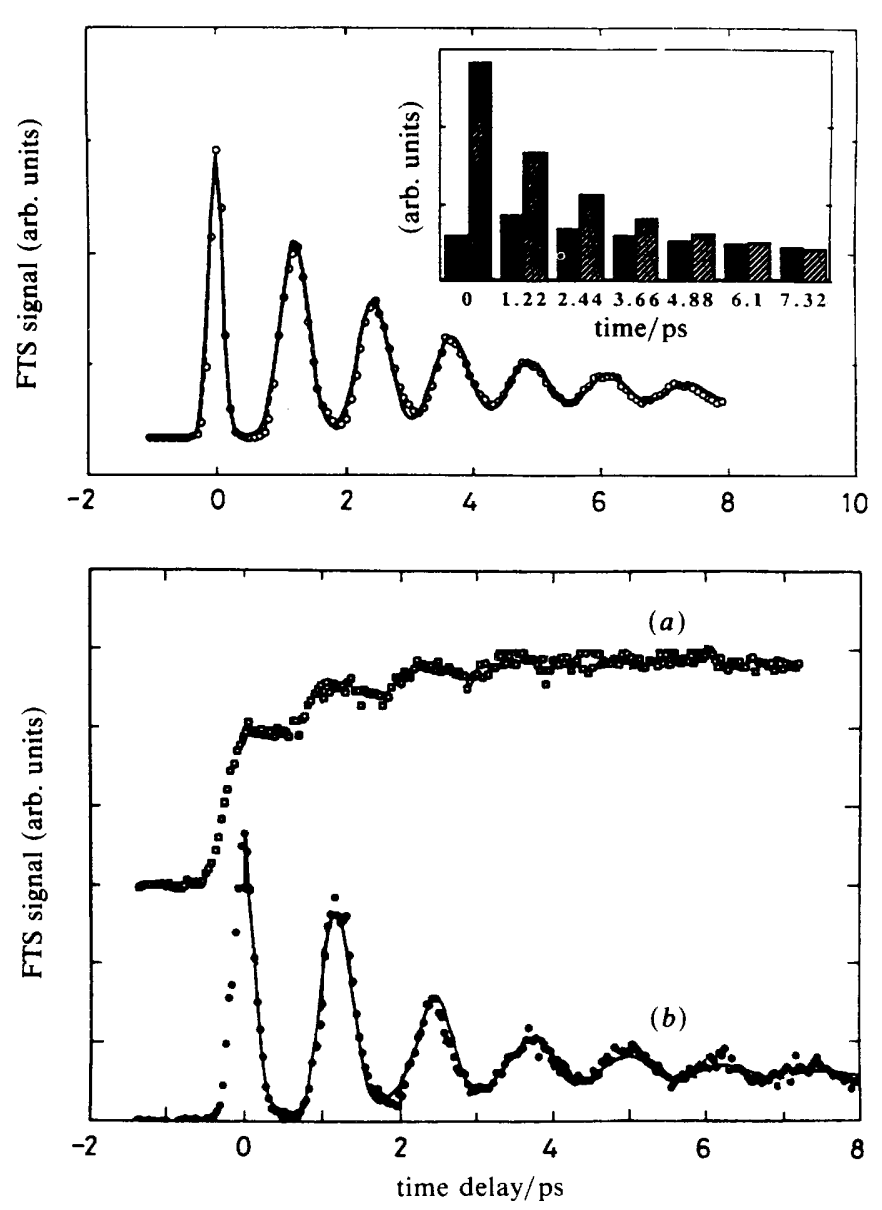

Fig. 2 Top: the oscillatory (resonance) behaviour observed by detecting the activated complex $[\mathrm{Na} \cdots \mathrm{I}] \#^{*}$ in the transition region. In the insert, both the peak area (solid) and peak height (hatched) of the oscillations are shown to indicate the leakage through the Landau-Zener region and the dephasing of the wave packet. Bottom: detection of free fragments $(a)$ and the activated complex $(b)$

of going out to form $M+X$ depends on the coupling between the two potential curves (a Landau-Zener type of interaction). Quantum-mechanically, this coupling changes the nature of the wavefunction as the internuclear separation, $R$, changes.

If we probe the motion in the transition region, $[\mathrm{M} \cdots \mathrm{X}] \ddagger^{*}$, we should observe the resonance motion between the covalent and ionic curves, with a damping determined by the leakage probability through the Landau-Zener region. On the other hand, if free $\mathbf{M}$ atoms are detected, one should see an increase in the number of $\mathbf{M}$ atoms every time the system executes a resonance period, i.e. a series of steps of increasing signal intensity determined by the period of covalent to ionic motion. Such observations (see e.g. Fig. 2) have been made by Rose et al. and Rosker et al. $^{6}$ and related to the resonance period, the lifetime of the activated complex, the escape probability and the magnitude of the coupling (covalent/ionic). [The $\Omega=1$ curve is a repulsive curve and has dynamical characteristics similar to other reactions studied by Dantus et al. and Rosker et al. (ICN), and by Glownia et al. and Bowman et al $\left(\mathrm{Bi}_{2}\right){ }^{8}{ }^{8}$ ] For example, at a total energy of $6850 \mathrm{~cm}^{-1}$ above the $\mathrm{Na}+\mathrm{I}$ channel, the period is $1.25 \mathrm{ps}$, the lifetime of the complex 

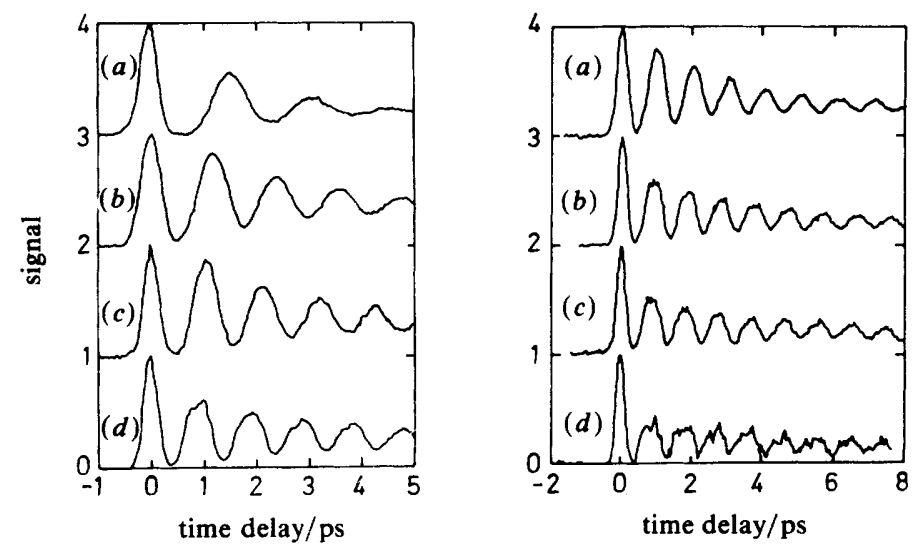

Fig. 3 The change in the period of the resonance as a function of total energy. Left: the wavelength $\lambda_{1}$ changes from (a) 300 , to $(b) 311$ to $(c) 321$ and to $(d) 339 \mathrm{~nm}$, respectively. (Right): $\lambda_{1}$ is (a) $332,(b) 344,(c) 354$ and (d) $364 \mathrm{~nm}$. The change of packet resonance frequency with energy over a wider range is given in ref. 6

is $34 \mathrm{fs}$, the recoil velocity is $0.029 \AA \mathrm{fs}^{-1}$ and the Landau-Zener escape probability is $18 \%$ (the largest value at this energy). The total splitting between the adiabatic curves at $R_{\mathrm{x}}$ is $0.1 \mathrm{eV}$. As discussed below, the wave packet spreads, and the spreading in time is larger than the lifetime of the complex; it can be observed experimentally (Fig. 2) because the probing window has sufficient $\Delta R$ resolution.

\subsection{The Two Potentials Involved}

In probing the transition region, we use laser-induced fluorescence and ionization/massspectrometric methods with a time delay resolution of $c a .10 \mathrm{fs}$. In principle, we have to consider the two potentials involved, the lower potential $V_{1}$ and the upper potential(s) $V_{2}$. As discussed by Bernstein et al., ${ }^{9}$ FTS enables determination of $V_{1}$ if $V_{2}$ is essentially flat at large $R$. In general, however, one probes $\Delta V(t)$, and this $\Delta V$ can be deciphered into $V_{1}(t)$ and $V_{2}(t)$ by changing the two wavelengths of the pump and probe, $\lambda_{1}$ and $\lambda_{2}$, in addition to scanning the delay time; i.e. measurements involve $I\left(\lambda_{1}, \lambda_{2} ; t\right)$.

In the centre-of-mass frame, $E=\frac{1}{2} \mu \nu_{r}^{2}$, and by changing $\lambda_{1}$, we can change $v_{r}$, the recoil velocity. Thus, for a fixed probe wavelength $\left(\lambda_{2}\right)$, the total energy can be changed systematically to observe the effect of $v_{r}$ on the dynamics of the motion between covalent and ionic curves. In Fig. 3 this behaviour is shown for NaI dissociation. Clearly, the potential is far from harmonic, and 'opens up' as we increase the total energy.

There is another measurement that helps us determine the nature of the $V_{1}$ potential. It is called a clocking experiment. ${ }^{7,10}$ If $t=0$ is determined precisely, then we can measure the time it takes the wavepacket to travel from $R_{0}$ at $t=0$ to $R$ of the probe. This time, $\tau$, must change depending on $E$ and the shape of $V_{1}$. As shown in Fig. 4, strongly repulsive $V_{1}$ and weakly repulsive $V_{1}$ have different temporal dynamics. For a fixed probe $\lambda_{2}$, we change the available energy ( $\lambda_{1}$ tuning) and determine clocking times $\tau(E)$. By self-consistency between the values of $\tau$ and $E$ we determine a unique length parameter $L$ for the repulsive branch [see eqn. (3)], and from the periods we determine the outer turning points at different values of $E$.

For illustration, we consider $R(t)$, from conservation of energy:

$$
R(t)=R_{0}+\int_{0}^{t}\left(\frac{2}{m}\left\{E-V_{1}\left[R\left(t^{\prime}\right)\right]\right\}\right)^{1 / 2} \mathrm{~d} t^{\prime}
$$



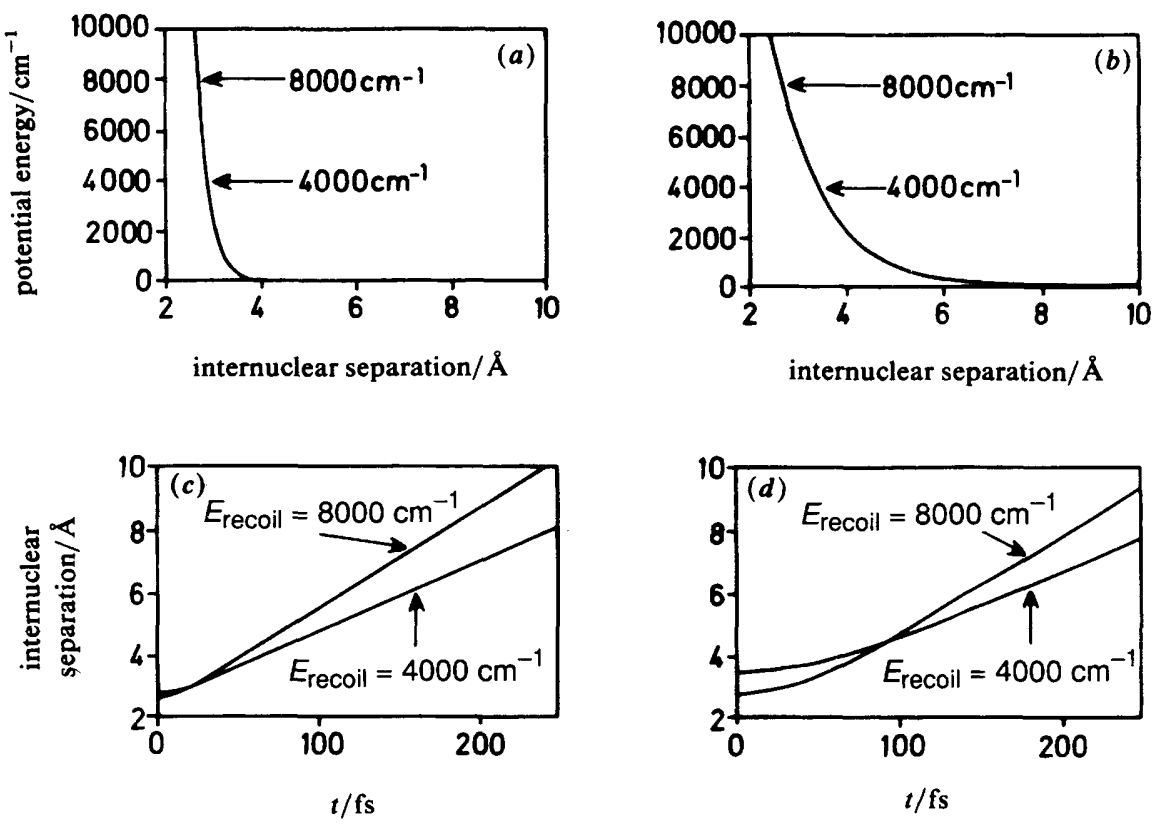

Fig. 4 The internuclear separation as a function of time, $R(t)$, at two different energies $[(c)$ and $(d)]$ and for the two different potentials $V(R)=V_{0} \exp \left[-\alpha\left(R-R_{0}\right)\right]$ in $(a)$ and $(b) . V_{0}=$ $8000 \mathrm{~cm}^{-1}, R_{0}=2.7 \AA, x=4.0 \AA^{-1}(a)$ and $1.0 \AA^{-1}(b)$

As an example, if

$$
V_{1}=A \exp \left(-\frac{\left(R-R_{0}\right)}{L}\right)
$$

then one can calculate the time it takes the particle to travel from $R_{0}$ to $R$. Solving the equation for $R(t)$, and taking $\Delta R \gg L$, one obtains

$$
\tau\left(R-R_{0}\right)=\frac{\left(R-R_{0}\right)}{v_{\mathrm{r}}}+\frac{L}{v_{\mathrm{r}}} \ln 4
$$

The first term describes the kinetic energy (velocity) effect; i.e. fragments begin at $R_{0}$ with the terminal velocity $v_{\mathrm{r}}$. The second term gives the influence of the exponent $L$ of the potential. As an example, for $L=0.4 \AA$ and $v_{\mathrm{r}}=0.018 \AA \mathrm{fs}^{-1}$ (total energy= $\left.2570 \mathrm{~cm}^{-1} ; 391 \mathrm{~nm}\right), \tau$ is $171 \mathrm{fs}$ for $R$ to change from 3.07 to $5.6 \AA$. This value is determined experimentally to be $175 \mathrm{fs}$, as discussed below. One can define a finite time for dissociation, $\tau_{\mathrm{d}}$, as the limit of the difference between $\tau$ and $\left(R-R_{0}\right) / v_{\mathrm{r}}$ and obtain the effect of $L$ on $\tau$, by analogy with particle physics definitions; for more discussion, see ref. 11.

The value of $\tau$ is different from a characteristic time, $\tau_{\frac{1}{2}}$, defined to include the window of the probe; during this time the potential drops to a value equal to $\gamma$ (the energy width of the probe window) the absorption of free fragment reaches half of its maximum value:

$$
\tau_{2}=\frac{L}{v_{\mathrm{r}}} \ln \frac{4 E}{\gamma}
$$

For the same $L, E$ and $v_{\mathrm{r}}$, mentioned above, $\tau_{\frac{1}{2}}=94 \mathrm{fs}$. This indicates that for this type of strongly repulsive $(L=0.4 \AA)$ branch, the potential drops to the $\gamma$-value and the 


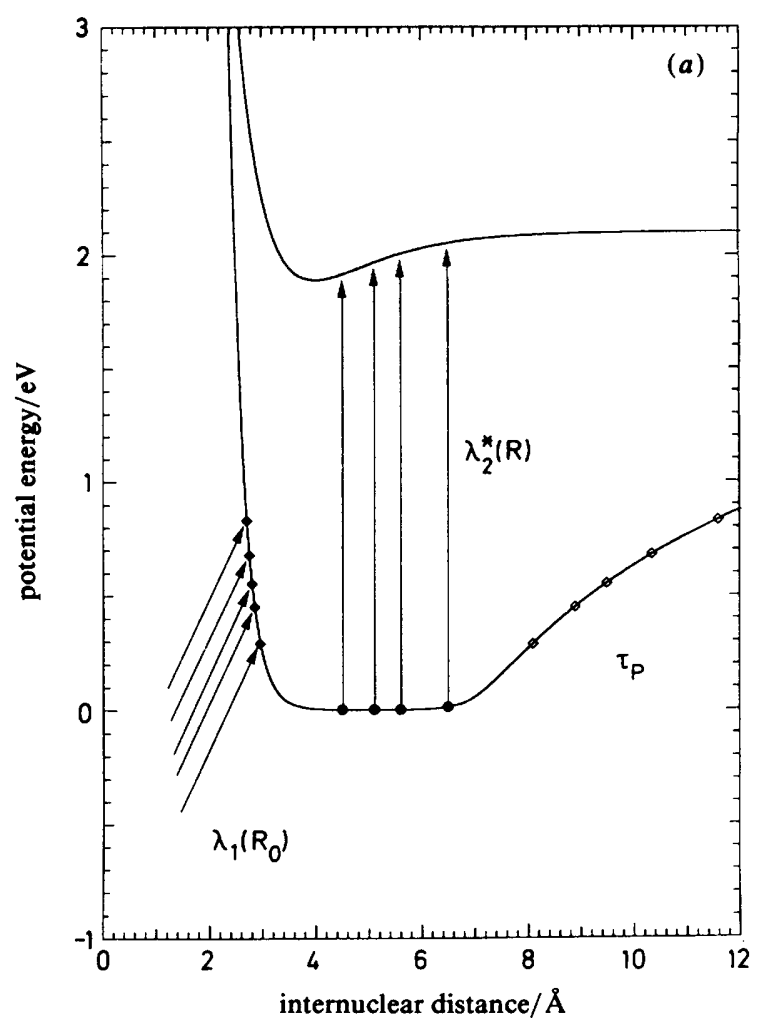

Fig. 5 (a) The PE curves of the NaI reaction and the experimental range of points reached in the FTS studies by changing the total energy $\lambda_{1}\left(R_{0}\right)$ and the probe position $\lambda_{2}^{*}(R)$, and by measurement of the period $\tau_{p}$. (b) and (c) The experimentally deduced $V_{1}(c)$ and $V_{2}(b)$. For $V_{2}$ there are two sets of data, using different ways of measuring the clocking times, and the results give the potential well discussed in text. The dotted curve in $(b)$ is the one deduced in ref. 12 . For $V_{1}$, the experimental points give the repulsive branch, with $L=0.4-0.5 \AA$, and the coulombic branch. The value of $0.4-0.5 \AA$ is consistent with other studies, ${ }^{64}$ but larger than the value of $0.245 \AA$ reported in ref. 65 . We have taken a simple repulsion form, eqn. (2), and this can be modified in form to include other terms (see ref. 66 and 67). The dotted line is the potential predicted by inversion of the data, and the circles give the RKR potential. ${ }^{13,66}$ The solid line is the potential from ref. 6 , which has been used in many calculations. These studies will be discussed and compared in more detail in ref. 68

absorption of free fragment (50\%) appears after $94 \mathrm{fs}$. In this $94 \mathrm{fs}, R$ is $4.2 \AA$. In the case of $\mathrm{NaI}$, there is an avoided crossing (not just a simple repulsive curve) and free fragments are born after the crossing point. Also, eqn. (4) assumes $V_{2}$ to be flat over the region of interest. The important point about this equation is that it describes the physics of the probing window and the role played by the weak dependence on $\gamma$ in defining the bond-breaking time, as discussed in ref. 7,9 and 10. Fig. 4 illustrates $R(t)$ $v s . t$ in two regimes, emphasizing velocity and distance effects on two different potentials, and the self-consistency between $R(t)$ and $E$ to determine $L$. The data obtained in Fig. 5 give $L=0.4-0.5 \AA$ for the repulsive branch and the $1 / R$ dependence for the coulombic branch. (A more precise determination of $L$ is currently underway.)

To probe $V_{2}$, we then fix the total energy and change $\lambda_{2}$ systematically. These experiments, which sweep the absorption (in time) from the free fragment to the transition region, give the nature of the upper potential reached by $\lambda_{2}$. In Fig. 5 we summarize 

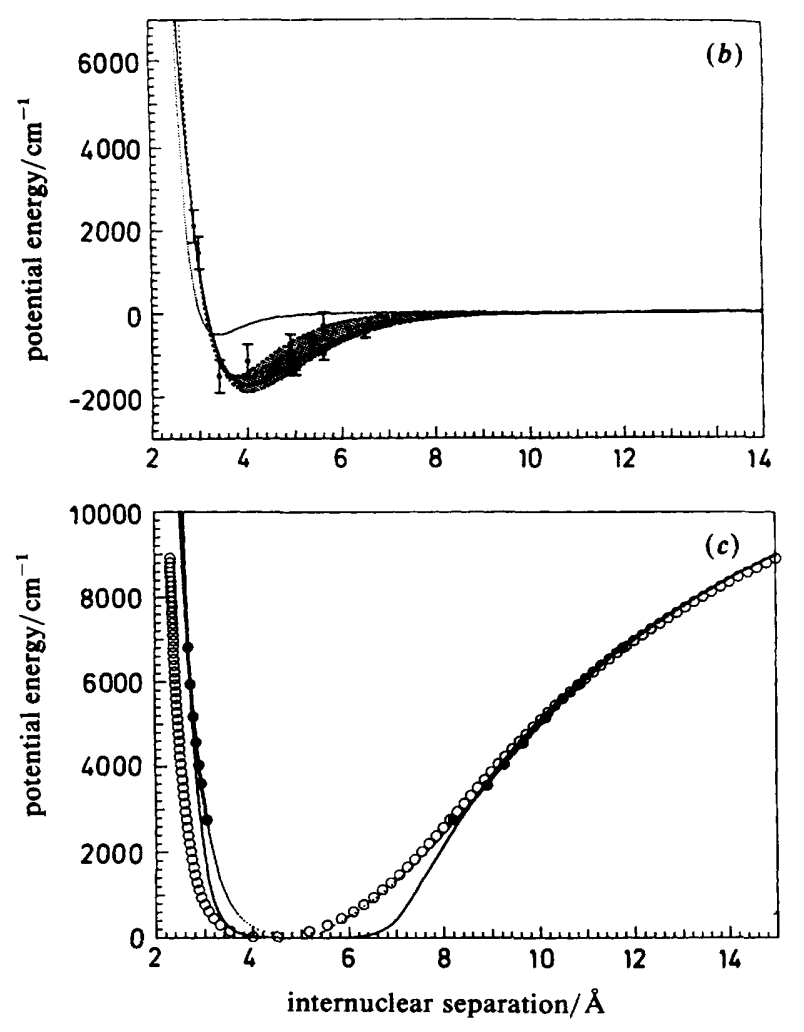

Fig. 5. (continued)

our findings for both $V_{1}$ and $V_{2}$, probed by FTS. The well found in $V_{2}$ is deeper ( $c a$. $0.2 \mathrm{eV})$ than that previously deduced $\left(530 \mathrm{~cm}^{-1}\right)$ by Bower et al., ${ }^{12}$ and is consistent with a recent spectroscopic study by Bluhm et al. ${ }^{13}$ For $V_{1}$, our probing distance of the potential is over the range of $R$ from $c a .2 .7$ to $12 \AA$.

\subsection{Dynamics of the Activated Complex Motion and Recurrences}

From the damping of the resonance oscillations, we obtained the coupling matrix element $\left(415 \mathrm{~cm}^{-1}\right)$ between the covalent/ionic curves, which is in good agreement with the theoretical calculation by Grice and Herschbach ${ }^{14}\left(400 \mathrm{~cm}^{-1}\right)$. From the period of resonance oscillations and the clocking times, we deduced the PES (Fig. 5) and the dynamics (Fig. 2 and 3). However, the quantum nature of wave-packet spreading with time can be obtained only if the window's resolution is sufficient to observe the coherent/incoherent changes in the motion.

For a free particle, a wave packet spreads appreciably for times given by

$$
\tau_{\mathrm{s}}=\frac{2 m}{\hbar}\left(\Delta R_{0}\right)^{2}
$$

showing the natural relationship to the mass, $m$. For NaI, $\tau_{\mathrm{s}} \approx 6 \mathrm{ps}$. Accordingly, a $1 \AA$ wave packet in $R$-space will begin to spread appreciably after 6 ps. Manifestations of this spreading come from the change of the width of the observed resonance with time (Fig. 2). Thus, both the spreading or dephasing of the wave packet and the population 

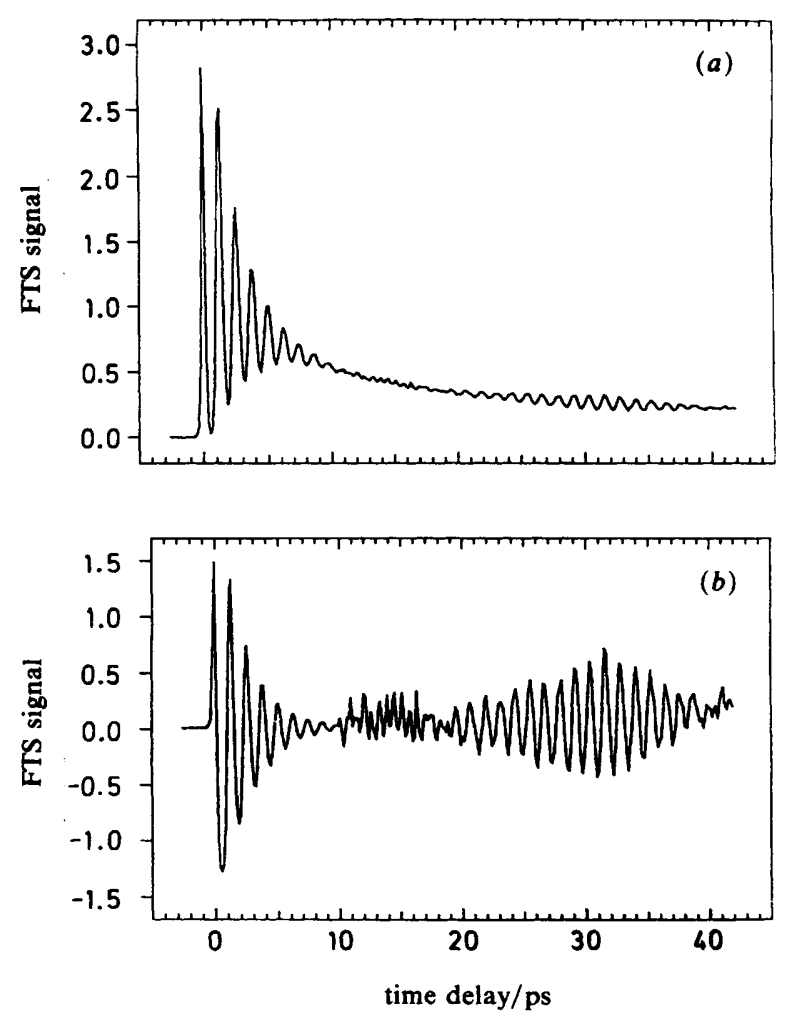

Fig. 6 (a) The oscillatory behaviour of the activated complex [ $\mathrm{Na} \cdots \mathrm{I}] \ddagger^{*}$ and the rephasing (echo) at long times, 30-40 ps. (b) The modulated part of the FTS signal, without the overall damping

leakage through the Landau-Zener region are present. Detection of free $\mathrm{Na}$ gives the population time constant(s), and in fact, as shown by Cong et al., ${ }^{15}$ the build-up of population is not a single exponential, but can be fitted to two exponentials. This non-exponentiality indicates that the packet is inhomogeneous in its level structure ${ }^{15}$ (see below). In some sense, this is reminiscent of $T_{1}$ - and $T_{2}$-type relaxations, an analogy which has been made clearly by Beswick and Jortner. ${ }^{16}$

Can isolated molecular systems dephase and then rephase again to give an echo? Because the wave packet executes the different resonances of the motion (or equivalently spans the different quasi-bound levels) owing to the avoided crossing, there are different timescales for the motion: the packet first oscillates between the covalent and ionic curves, and spreads on the picosecond, not femtosecond, timescale. In the process of breaking the bond, the packet spreads significantly, and at $c a .15 \mathrm{ps}$ our results indicate that it becomes so-called 'chaotic', spreading in all regions of $R$. Calculations of snapshots at these times confirm this picture.

By accident, we discovered that if we wait long enough (30-40 ps), this so-called 'chaotic' packet appearance starts to take on a coherent appearance, and recurrence echo of the initial resonance oscillations (with the same period!) occurs (Fig. 6). These recurrences are very sensitive to the total available energy, and reflect the nature of the PES caused by the avoided crossing. The observations represented our first example of seeing the wavefunction of an isolated molecular system, in the course of the reaction, spreading and rephasing again. This brings to focus another point. Even though the wave packet spreads and appears 'chaotic', the system is actually non-chaotic; the wave 


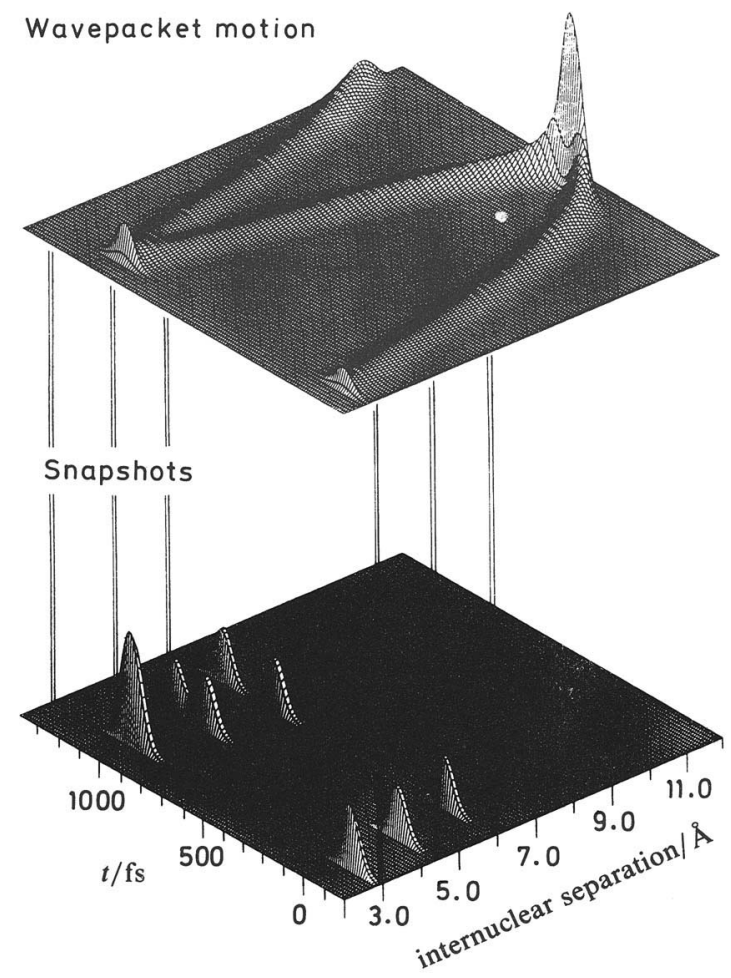

Fig. 7 Simulation of the wave packet motion in 2-D space of $R$ and $t$. The lower plot shows the snapshots of the wavepacket motion at different internuclear separations. Note the time evolution of the splittings as $R$ increases

nature and the phases of the individual wavefunctions constituting the packet are still well defined, up to $40 \mathrm{ps}$; otherwise recurrences would not have occurred!

\subsection{Observation of the Trajectories in $R$ and $t$}

Fig. 7 displays trajectories in the $R v s$. $t$ domain. As shown in the figure, the wave packet changes direction as $R$ and $t$ change, starting from the initial time zero at $R \approx 2.7 \AA$. After half a period, the packet has reached the ionic turning point, and after a complete period, it has returned to the covalent turning point. At or near the ionic-covalent crossing, the packet bifurcates; some of it continues to move in the direction of increasing $R$, becoming $\mathrm{Na}$ and $\mathrm{I}$, and the rest remains as $[\mathrm{Na} \cdots \mathrm{I}]$ †* $^{*}$, oscillating between the covalent and ionic curves (see also Fig. 1).

To observe the motion of the trajectories, a window in $R(t)$ must be opened with sufficient distance resolution $\Delta R$ to allow us to view the directionality of the packet at different times. If the window is stepped continuously along $R$, then we can map out the motion and characteristics of the PES. For a given distance resolution, the temporal motion represents a snapshot of the PES at this particular $R$. For example, a window at $R=3 \AA$ will give the snapshot shown at the bottom of Fig. 7, which is basically the oscillatory motion displayed in Fig. 2. On the other hand, if a snapshot is taken at $R=4.5 \AA$, the oscillatory motion displays a splitting because at this distance the packet is going in and out of the probing $\Delta R$ window. At longer $R$, the splitting increases in time, because the distance the trajectory travels is longer. The observation of such 

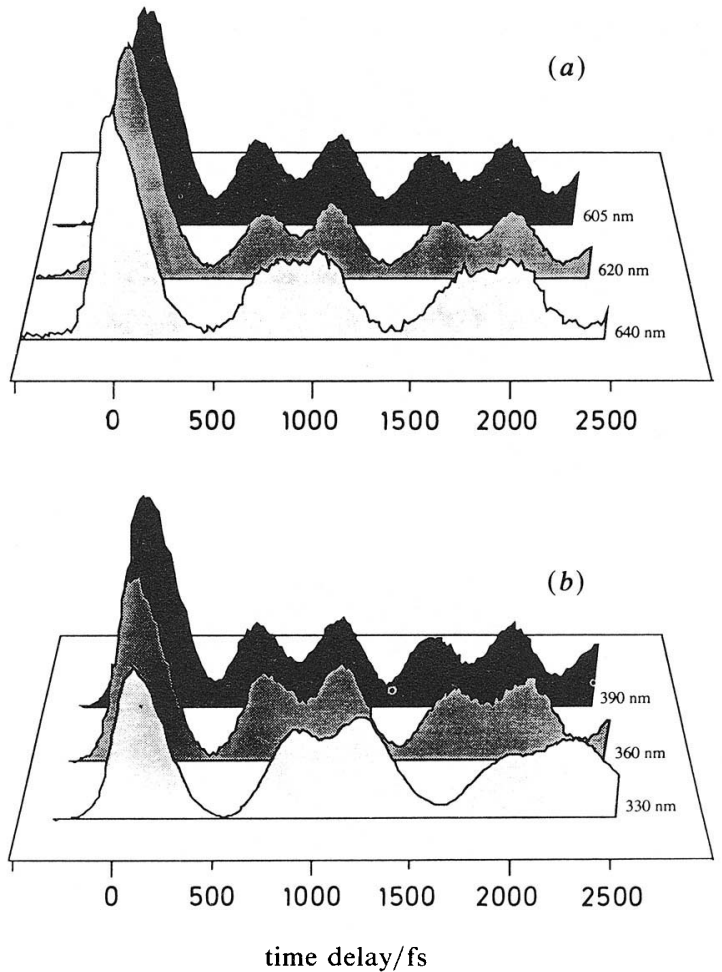

Fig. 8 Experimental femtosecond snapshots of the NaI reaction. (a) shows the snapshots taken at different $\lambda_{2}$, while maintaining the total energy fixed. $(b)$ shows the reverse

splitting would indicate two important points. First, it would mean that the window opened in $R$ is sufficiently narrow to resolve the nuclear motion in and out of that region of the PES. Secondly, the splitting gives a time (at each $R$ ) that is directly related to the clocking time, defined by the distance travelled from time zero to the probing point on the PES, as discussed before.

Fig. 8 shows the experimental observations by Mokhtari et al. ${ }^{17}$ for the NaI reaction. The packet was prepared by a femtosecond pulse at a wavelength $\lambda_{1}$ corresponding to $E \approx 2570 \mathrm{~cm}^{-1}$ above the $\mathrm{Na}+\mathrm{I}$ threshold. To probe at different $R$, we used another femtosecond pulse of a different wavelength $\lambda_{2}$. For a given total energy in the bond, both the time and $\lambda_{2}$ can be changed systematically to obtain the snapshots at different values of $R$. Three snapshots in Fig. 8 are shown as examples of the many results obtained. The evolution of the splitting as $\lambda_{2}$ decreases (i.e. as $R$ increases) is evident. The splitting at $\lambda_{2}=620 \mathrm{~nm}$, for example, is $350 \mathrm{fs}$. This gives a clocking time for the packet to reach the probe on the PES of $175 \mathrm{fs}$. The distance being probed is $5.6 \AA$, and this is consistent with theory, as discussed above. The window resolution is very appropriate for studying the dynamical motion of chemical reactions: the temporal splitting observed gives an experimental $\Delta R \approx 0.5 \AA$ on the PES. The potential for these types of reactions is quite flat (non-bonding type) at intermediate $R$ (Fig. 1), and therefore even better resolution may be achieved in other systems.

As $E$ is decreased, we expect the velocity to decrease and the distance travelled by the wave packet, to a given $R$, to decrease also. Depending on the PES, these two effects determine whether or not the splitting will increase or decrease with $E$ (Fig. 4). Fig. 8 shows three snapshots at different $\lambda_{1}$. We observe an increase in the splitting as $E$ is 
lowered. We also observe a decrease in the period (Fig. 3). The latter is consistent with the potential 'narrowing' in $R$ at lower $E$ (Fig. 5). The former indicates that the covalent side of the potential is very repulsive at short $R$ and is dominated by velocity (not distance) effects (Fig. 4), as deduced in Fig. 5, and discussed above.

\subsection{Theory}

Since the early report of these experiments, all major features have been understood using an intuitive classical mechanical description. Because the system is relatively heavy and we are interested in the early time motion, the centre of the wave packet is described as particles moving according to the laws of classical mechanics. Relying on these ideas makes the visualization of the experiments very simple.

However, the wave properties of the particle are manifested in several ways. First, the spreading of the wave packet, which as shown above for NaI, is beginning to be appreciable at $c a .6 \mathrm{ps}$ (for an electron, in contrast, this time is $c a \cdot 10^{-16} \mathrm{~s}$ ). Secondly, the uncertainty principle for a free wave packet

$$
\overline{\Delta R^{2}} \overline{\Delta P^{2}} \geq \hbar^{2} / 4
$$

indicates that a wave packet broad in $P$-space is narrow in $R$-space. For heavy particles, the de Broglie wavelength is sufficiently small that wave properties may appear to be unimportant; for $\mathrm{NaI}$, the de Broglie wavelength is $c a .0 .2 \AA$, compared to a distance motion of $\geqslant 7 \AA$. Thirdly, the wave packet at $t=0$ is not the simple 'classical mechanical packet' projected from the ground state. The packet shape must take into account the question of the preparation by a finite pulse width: the classical picture is equivalent to a preparation by a pulse of zero duration (such pulses have been discussed ${ }^{18}$ recently!). Finally, classical mechanics fails to describe the spread and the (non-chaotic) rephasing of coherence particularly at long times, up to $40 \mathrm{ps}$, unless one designs different initial trajectories for simulations.

Classical mechanical theory ${ }^{9,11}$ has reproduced the oscillatory motion ${ }^{6,19}$ using the known PES and the equation of motion of $R(t)$. It also shows the salient features of many experimental observations, as discussed by Rosker et al., ${ }^{6,7}$ Bersohn et al. ${ }^{11}$ and Lee et al. ${ }^{19}$ Semiclassical treatments by Marcus ${ }^{20}$ and by Lin and Fain ${ }^{21}$ have also been successful in obtaining the early-time oscillatory motion and damping.

Metiu and co-workers ${ }^{22}$ have shown that rigorous quantum calculations are able to describe the FTS observations from $t=0$ to $c a .8 \mathrm{ps}$. These studies are important in showing that quantum calculations, involving all known (not adjustable) parameters of the PES and the preparation process, are in accord with experiments. The calculations also illustrate the presence of spreading (less than observed, however) and show splittings on the peaks which were not identified with the clocking of the reaction. Snapshots of the packet at different times indicate the motion between the covalent and ionic regions. The quantum calculation of Choi and Light ${ }^{23}$ has successfully reproduced the oscillatory behaviour of the motion. All of these calculations describe the global dynamics in the region $0-10$ ps.

Chapman and Child, ${ }^{24}$ in a beautiful paper, showed that the recurrences at long times are manifestations of the distribution of the level structure and resonance lifetimes (caused by the avoided crossing), and hence obtained the timescale for rephasing. Their calculations are in good agreement with experimental observations at long times, 30$40 \mathrm{ps}$. In ref. 15 we discuss this in relation to the dynamics of the packet in the adiabatic well and its interaction with the ground state.

Very recently, Fujimura and Kono ${ }^{25}$ have computed the motion in the $R$ and $t$ domain and showed the trajectory of the motion at different total energies. They obtained splittings in the FTS resonance, similar to those shown in Fig. 7. Quantification of the 
theoretical results with the PES derived from FTS experiments will be given in detail in ref. 68.

\subsection{Summary}

The results reported here illustrate the concepts and methodology involved in probing the dynamics on the femtosecond timescale. The motion of the nuclear wave packet during the breaking of the $\mathrm{NaI}$ bond can be observed with an experimental resolution of $c a .0 .5 \AA$ of internuclear separation. The total energy and the position of the probing 'window' can be varied to map the trajectories $R(t)$ and the shape of the potentials involved. The range of internuclear distances probed is up to $6.5 \AA$ on the covalent and $12 \AA$ on the ionic curves. The dynamics probed in real time include motion of the activated complex, its damping by the avoided crossing between covalent and ionic potentials, and quantum spreading and rephasing of the wave packet as the complex decays to final fragments. Theory is quite advanced for these systems, and provides, successfully, the global picture.

Because of their 1-D nature, these alkali halide reactions serve as prototype systems for studying simple elementary reactions and they help in understanding the concepts involved. But what about motion in two (or more) dimensions?

\section{Probing the Transition-state and Dynamical Resonances}

In the case of alkali halides, the activated complex ${ }^{5}$ spans a configuration defined by the separation of atoms in the $R$-region (one coordinate). In the full collision along the covalent curve, reaction (II),

$$
\mathrm{Na}+\mathrm{I} \rightarrow[\mathrm{Na} \cdots \mathrm{I}] \ddagger^{*} \rightarrow \mathrm{Na}+\mathrm{I}
$$

the complex path is continuous along a repulsive curve. However, when the activated complex is prepared at a specified configuration in a half-collision, $[\mathrm{Na} \cdots \mathrm{I}] \ddagger^{*}$ is established at a given $R$, and its dynamics can be followed. There is, of course, no saddle point in this case.

If the reaction dynamics involve more than one coordinate, an interesting question arises: can one observe in real time the reactive evolution from the TS at the saddle point to final products on the global PES? The simplest system for addressing the question is of the type $\mathrm{ABA} \rightarrow \mathrm{AB}+\mathrm{A}$. This system is the half-collision of the fullcollision

$$
\mathrm{A}+\mathrm{BA} \rightarrow[\mathrm{ABA}] \ddagger \rightarrow \mathrm{AB}+\mathrm{A}
$$

It involves one symmetrical stretch $\left(Q_{1}\right)$, one antisymmetrical stretch $\left(Q_{2}\right)$, and one bend $(q)$.

We have chosen the system $[\mathrm{I} \cdots \mathrm{Hg} \cdots \mathrm{I}] \ddagger^{*}$, for which the antisymmetric (translational) motion gives rise to vibrationally cold (or hot) nascent $\mathrm{HgI}$. The symmetric motion is bound and may give rise to a dynamical resonance. The idea of the experiments was to excite directly the collision complex from the ground state to the transition state, $[\mathrm{I} \cdots \mathrm{Hg} \cdots \mathrm{I}] \ddagger^{*}$, and to follow the real-time dynamics of the complex as the motion proceeds from $t=0$ (transition state) to long times (free fragments). The energetics are shown in Fig. 9. One advantage of exciting the complex directly is the possibility of observing the dynamics with minimum-impact parameter averaging. In the full collision case, this averaging may 'smear out' dynamical resonance effects. The concept of transition-state detection along symmetric and antisymmetric coordinates is illustrated using the PES of Fig. 10 in these types of reactions.

The dynamics of the complex in this case is fundamental to the understanding of the TS of reactions involving more than one degree of freedom, and, as pointed out by 


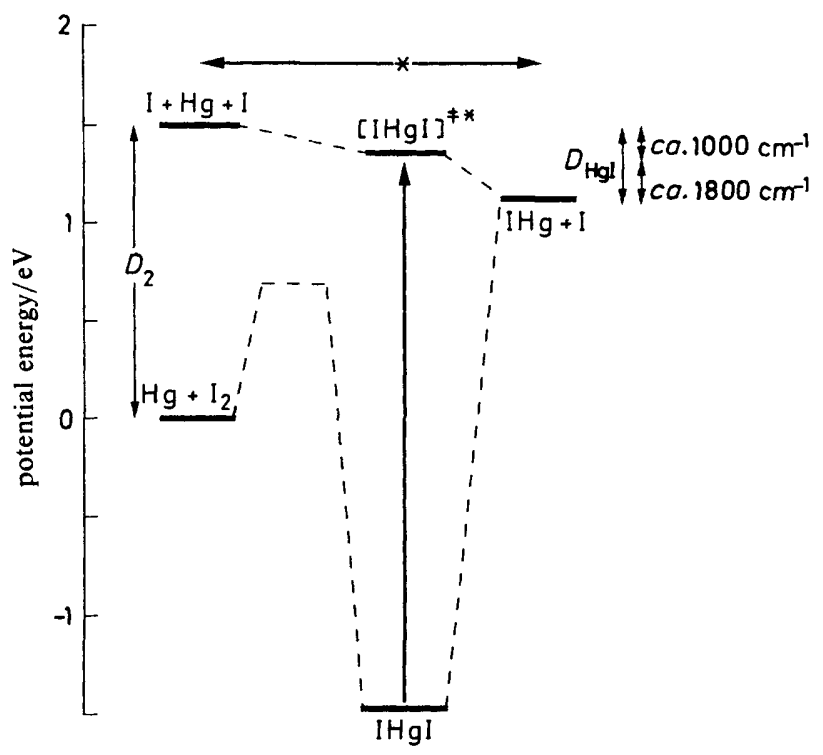

Fig. 9 Energetics for the reaction of $\mathrm{Hg}+\mathrm{I}_{2}$, and excitation of the $\mathrm{TS},[\mathrm{I} \cdots \mathrm{Hg} \cdots \mathrm{I}] \neq^{*}$ (for the ground state surface, see ref. 69). The star indicates an arbitrary excitation where both channels are open

Bernstein, ${ }^{26}$ it is directly connected to the bimolecular case of $\mathrm{Hg}+\mathrm{I}_{2}$. In theory, ${ }^{27}$ one projects the initial wavefunction onto the final product-state wavefunction and obtains expressions for product-state distributions. If, however, the wave-packet motion is described along $Q_{1}$ and $Q_{2}$, one considers the structural and vibrational frequency changes on going from the transition-state region to the final products. It is therefore of great interest to attempt probing such motion in this class of reactions in real time, and to relate the observables to the nature of the force field along the reaction coordinate.

There are a number of interesting features for this reaction. First, the equivalence of the two bonds makes the PES symmetrical with respect to the $\mathrm{I}, \mathrm{HgI}$ coordinate and the reaction path is well defined along the antisymmetric coordinate (Fig. 11). Secondly, because of the large change in structure, one expects changes in the vibrational frequencies on going from the transition-state region to the final products. The $A-B$ bond in the upper state of ABA corresponds to a vibrationally excited A-B fragment. The bend gives rise to rotations. Thus, the dissociation impulse produces $\mathrm{HgI}$ in different vibrational/rotational states allowing one to monitor different sets of reaction trajectories.

\subsection{Observation of the TS and Nascent Fragments}

The TS was prepared by a laser pulse at $\lambda_{1}=310 \mathrm{~nm}$. This amounts to $c a .12500 \mathrm{~cm}^{-1}$ of energy available for vibration and translation in the $\mathrm{HgI}+\mathrm{I}$ channel, as discussed by Dantus $e$ t al. ${ }^{28}$ and shown in Fig. 9 and 12. As the $\mathrm{HgI}$ and $\mathrm{I} / \mathrm{I}^{*}$ (or $\mathrm{Hg}+2 \mathrm{I}$ ) fragments separate, they are probed by a second laser pulse at a different $\lambda_{2}, e . g .620$ or $390 \mathrm{~nm}$. At short interfragment distances, the separation between the PE surfaces leading to $\mathrm{HgI}$ product in its $\mathrm{X}^{2} \Sigma^{+}$and $\mathrm{B}^{2} \Sigma^{+}$states is smaller than in the asymptotic limit. The $620 \mathrm{~nm}$ pulse thus probes early times, and $390 \mathrm{~nm}$ the long-time behaviour of the reaction.

Bowman et al. and Dantus et $a l^{28}$ studied FTS of the reaction and observed the femtosecond transients of the TS and the nascent fragments. Fig. 12 shows transients probed at $390 \mathrm{~nm}$, differing only in the detection wavelength $\lambda_{\text {det }}$, which probes different $\left\langle v^{\prime \prime}\right\rangle$ levels in the $\mathrm{X}$ state of $\mathrm{HgI}$ (Fig. 13). They are very dissimilar in characteristics, 

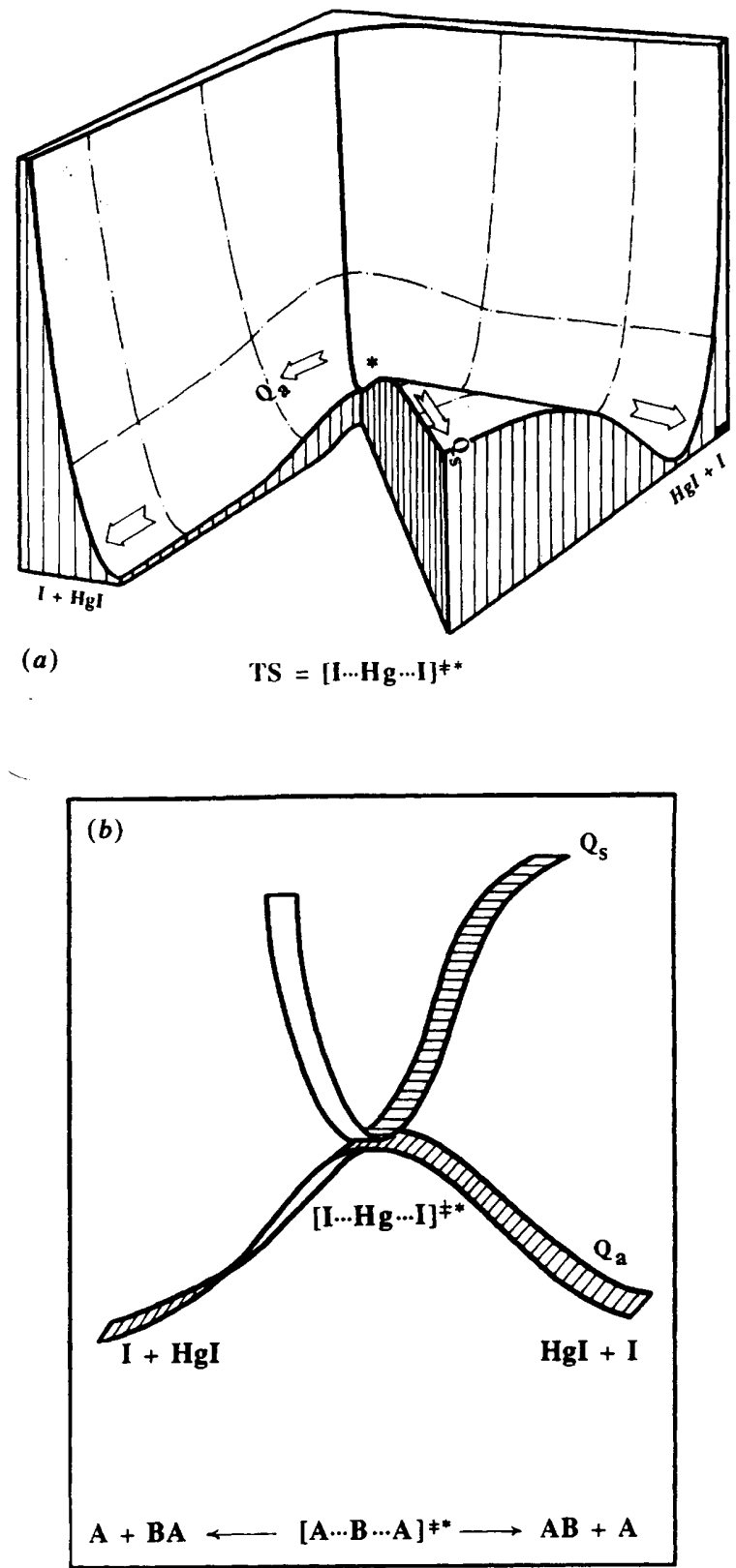

Fig. 10 (a) The PES and the transition state for the $[\mathrm{I} \cdots \mathrm{Hg} \cdots \mathrm{I}]$ ** $^{*}$ system. (b) A (schematic) cut along the symmetric and antisymmetric coordinates

even when detected at nearby wavelengths (e.g. $\lambda_{\text {det }}=427.5$ and $432.5 \mathrm{~nm}$ ). Fig. $12(a)$ shows rapid oscillations (with a period of $300 \mathrm{fs}$ at longer delay times) observed at $\lambda_{\text {det }}=427.5 \mathrm{~nm}$, corresponding to $\mathrm{HgI}$ vibrational motion near the bottom of the anharmonic potential well, while Fig. 12(b) shows an initial sharp peak followed by slow

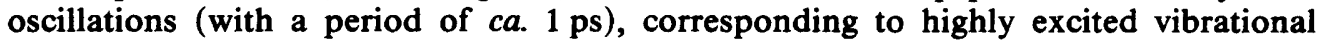
motion of $\mathrm{HgI}$ near the dissociation limit (Fig. 13). 


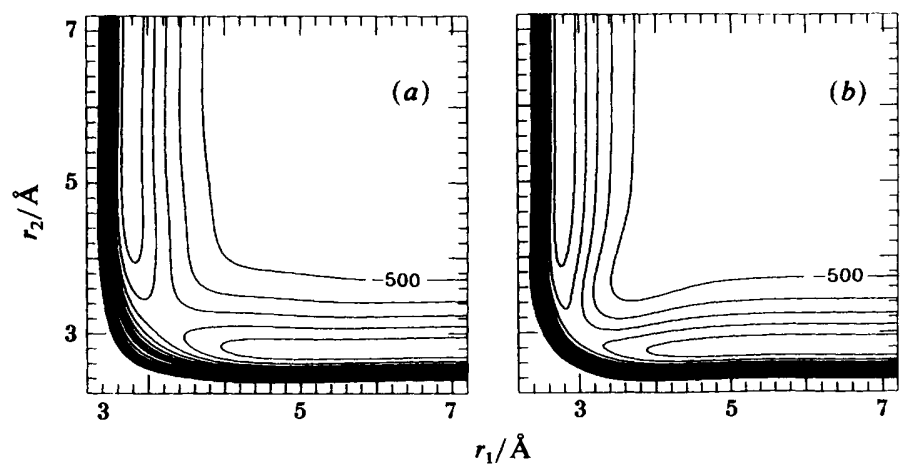

Fig. 11 The two types of PES $[(a)$ and $(b)]$ used in the theoretical calculations of wave-packet propagation from the TS to free fragments (for the parameters, see ref. 35)
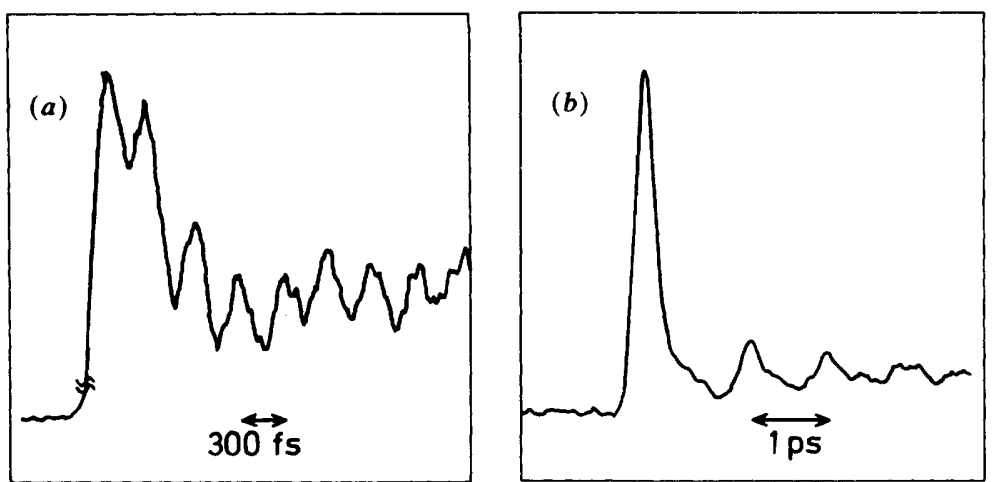

Fig. 12 Experimental FTS displaying the transitory behaviour observed in the detection of nascent $\mathrm{HgI}$ in different $v^{\prime \prime}$ states as it evolves from the TS: (a) $E_{\mathrm{Hgl}, \mathrm{l}}=4900 \mathrm{~cm}^{-1},\left\langle v_{\mathrm{Hgl}}^{\prime \prime}\right\rangle=7 ;(b)$ $E_{\mathrm{Hgl}, \mathrm{I}}=12500 \mathrm{~cm}^{-1},\left\langle v_{\mathrm{Hgl}}^{\prime \prime}\right\rangle=29$. The total available energy for product vibration and translation was the same $\left(12500 \mathrm{~cm}^{-1}\right)$. The detection method identifies the $I$ and $I^{*}$ channels, and hence the energy available in $(a)$ (the $\mathrm{I}^{*}$ channel) is less by $7600 \mathrm{~cm}^{-1}$ (see text and ref. 28). Note the drastic change in the oscillatory behaviour with time and energy

In the $I^{*}$ channel, relatively less energy is available for vibrational and translational excitation, having mostly been taken up by spin-orbit excitation of I atoms. Ground-state $\mathrm{HgI}\left(\mathrm{X}^{2} \Sigma^{+}\right)$is formed in low vibrational levels, then probed to the $\mathrm{B}^{2} \Sigma^{+}$state. The resulting levels fluoresce strongly at $427.5 \mathrm{~nm}$, less at $400 \mathrm{~nm}$, and very little at 432.5 or $360 \mathrm{~nm}$ compared with the I channel. In the I channel, highly vibrationally excited $\mathrm{X}^{2} \Sigma^{+}$product is formed and probed to a different set of $\mathrm{B}^{2} \Sigma^{+}$state vibrational levels; these fluoresce strongly at 432.5 and $360 \mathrm{~nm}$, less at $440 \mathrm{~nm}$, and little at $427.5 \mathrm{~nm}$, relative to the $I^{*}$ channel. These fluorescence spectra thus allow a selection of the dynamics to be probed.

For TS detection, we kept the total energy constant and changed the probe wavelength to a region where there is no absorption by free fragments (at $\lambda_{2}=620 \mathrm{~nm}$ ). The transients are shown in Fig. 14. We observe only two peaks, separated by $200 \mathrm{fs}$, and a fast damping imaging the ultrafast motion of the packet away from the saddle point toward final products.

The transients of the TS and nascent fragments in real time reflect the dynamics quite well. Vibrationally excited $\mathrm{HgI}$ is coherently formed in the decay of the complex, 
(a)

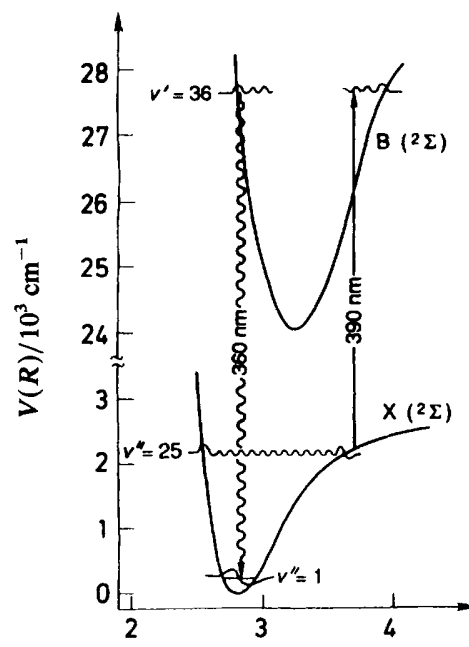

(b)

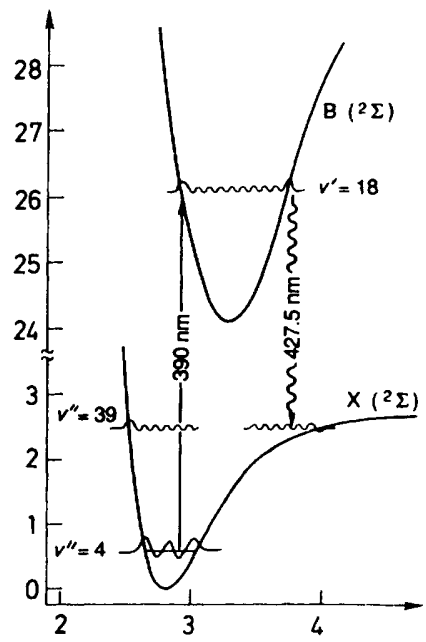

$R / \AA$

Fig. 13 The scheme used in the LIF to detect different $v^{\prime \prime}$ states of nascent product, $\operatorname{HgI}\left(v^{\prime \prime}\right)$

and the experiments probe different trajectories of the reaction to various final $v^{\prime \prime}$ states of $\mathrm{HgI}$, spanning the $v^{\prime \prime}$ distribution. This is evident in the change of the vibrational period observed for $\mathrm{HgI}$ with $\left\langle v^{\prime \prime}\right\rangle=7$ vs. $\left\langle v^{\prime \prime}\right\rangle=29$ in Fig. 12. The periods (at long delay times) are in good agreement with the spectroscopic analysis for stable $\mathbf{H g I},{ }^{29} \dagger$ and the delay of the signal gives the dissociation time, describing the change of the complex from $R_{0}(t=0)$ to the distance projected by the window of the probe. The observation of the TS describes the early-time motion in the symmetric-stretch direction, as discussed below.

\subsection{Time-dependent Anisotropy and the Geometry of the TS}

The above results give the temporal picture imaging the vibrational dynamics of the complex and fragment, and the electronic excitation of iodine (spin-orbit states). The bend motion is on a longer timescale, and has important consequences for the alignment. From time-integrated alignment experiments ${ }^{30}$ one knows the importance of the anisotropy in understanding the vectorial properties of the dissociation. Alignment in real time provides information on rotations, ${ }^{2,31}$ and more recently we have used it to study rotational (torque) dynamics in dissociation reactions. ${ }^{32}$ Here the experiments probe the relative alignment of the fragment transition moment and the initial alignment of the complex as a function of time. As shown in Fig. 15, the transients observed for the pump and probe in parallel polarization are very different, in their decay and buildup, from that of pump and probe in perpendicular polarization. Yet the vibrational periods observed in both transients are identical. The experiments indicate that while $\mathrm{HgI}$ is executing the vibrational motion, the torque on $\mathrm{HgI}$ is inducing rotations, and as a function of time the anisotropy changes. From these experiments, one obtains, in a simple description, the $\langle J\rangle$ produced as a result of the torque, the coherence time and 
(A)

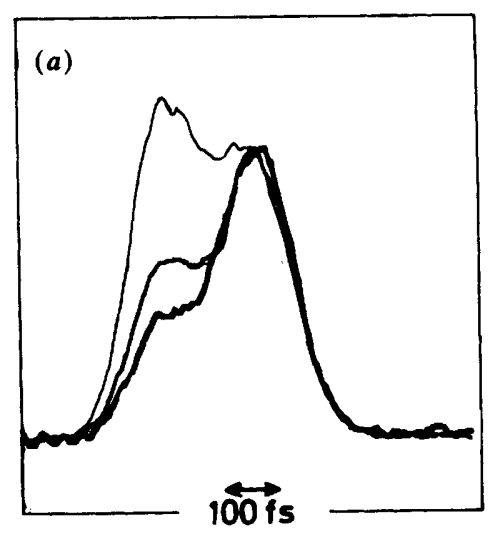

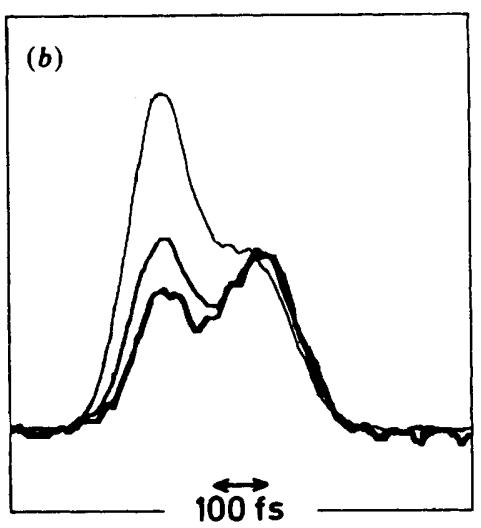

(B)

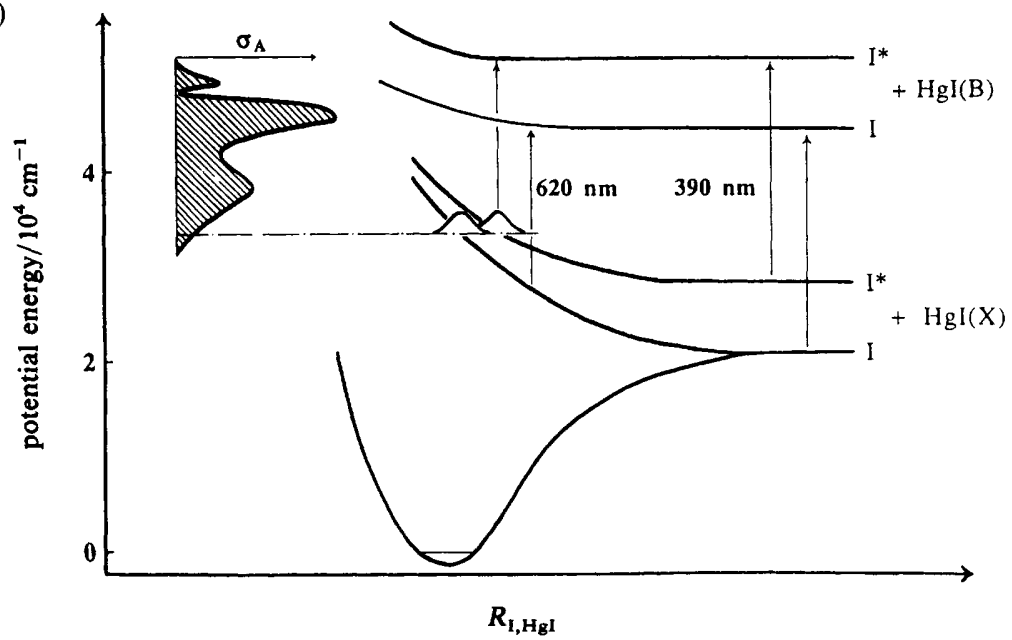

Fig. 14 (A) Transition-state femtosecond transients, exciting [I $\cdots \mathrm{Hg} \cdots \mathrm{I}] \ddagger^{*}$ at $\lambda_{1}=310 \mathrm{~nm}$ and detecting at $620 \mathrm{~nm}$. The transients in $(a)$ and $(b)$ are the same, but for $(a)$ parallel and (b) perpendicular polarization. The three transients in each panel are for different detection wavelength (thin line, $440 \mathrm{~nm}$; medium line, $390 \mathrm{~nm}$; and heavy line, $360 \mathrm{~nm}$ ). Note the $c a .200 \mathrm{fs}$ time spacing between the peaks, independent of polarization and emission detection wavelength. (B) A cut along the translational coordinate, together with the absorption cross-section, showing the idea for detection of the TS and nascent fragments

the geometry of the TS. There is a finite time for $J$ to become well defined, and this has been discussed elsewhere. ${ }^{32}$

The alignment ${ }^{30}$ is related to rotational angular momenta by ${ }^{32}$

$$
I(t)=C[1+\alpha r(t)] A(t)
$$

where $C$ and $\alpha$ are well defined constants, $A(t)$ is the scalar dissociation temporal function and $r(J, t)$ is the anisotropy function:

$$
r(J, t)=0.4\left\langle P_{2}[\cos \eta(t)]\right\rangle
$$

where $\eta$ is the time-dependent angle between the transition dipole of the pump transition at $t=0$ and that of the probe at time $t$. The theoretical fits in Fig. 15 were obtained when $J_{\max }=80$. The rotational distribution $(\Delta J)$ gives rise to the early time decay (and buildup) and defines a coherence time for rotational dephasing, measured to be $c a .1 \mathrm{ps}$. 


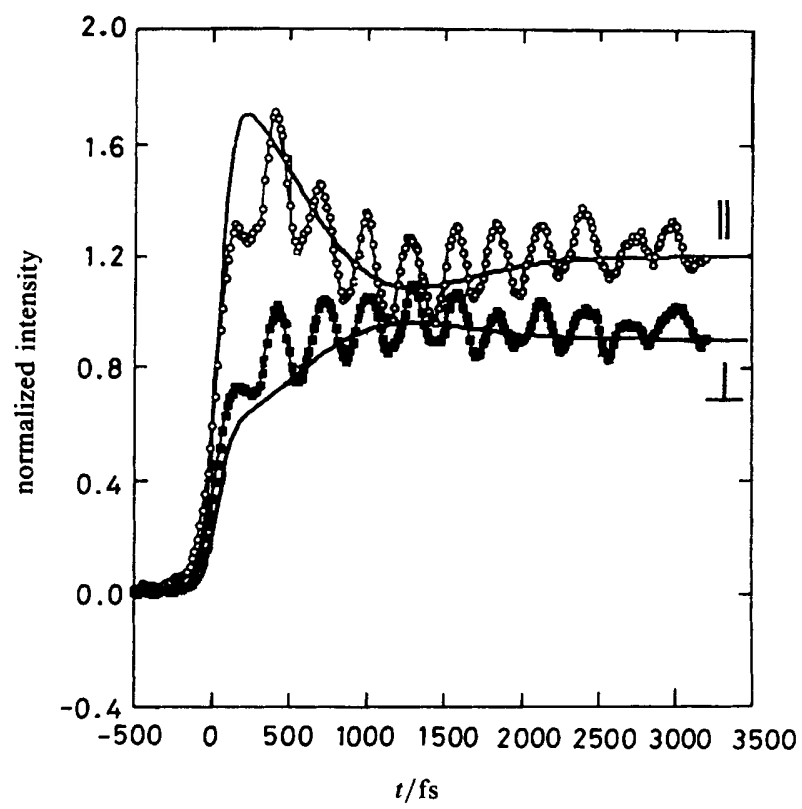

Fig. 15 The polarization anisotropy in real time. Note the build-up and decay, and persistence of the vibrational oscillation with the same phase in both 'parallel' and 'perpendicular' FTS experiments. The solid lines are the theoretical curves (see text)

The coherence time is longer than the time constant for scalar dynamics (dissociation of the complex). The $\mathrm{HgI}$ fragment rotates relatively slowly, $\omega_{0}=0.01 \mathrm{ps}^{-1}$. The dip observed in the transient of Fig. 15 reflects the shift of the rotational distribution from $J=0$, as detailed in Ref. 32. These polarization experiments complement the 'scalar experiments' in that $r(t)$ has signatures of the rotational and torque dynamics. It is interesting to note that we can now define $\tau \neq$ (complex dissociation time) and $\tau_{\mathrm{R}}$ (rotational), and these are the same quantities used by Herschbach to define direct and complex-mode reactions. ${ }^{33}$

\subsection{TS and the Dynamical Resonance}

Even without detailed theoretical calculations, the above understanding of the dynamics is intuitive, and is based on simple models for rotational and vibrational excitation, and on Polanyi's general concepts regarding the position of the TS on the PES. ${ }^{34}$ The analysis was verified in our earlier work ${ }^{28}$ by a two-dimensional classical trajectory calculation, which yielded dissociation times and vibrational distributions in agreement with experiment. The quantum simulation of the dynamics allowed a more detailed comparison with the FTS transients, as well as pointing to a number of interesting features of the reaction dynamics and $\mathrm{PES}$, including the early-time motion along the symmetric coordinate.

The theory has been detailed for the case of $[\mathrm{I} \cdots \mathrm{Hg} \cdots \mathrm{I}] \ddagger^{*}$ elsewhere, ${ }^{35}$ and the approach is that of a wave-packet analysis. ${ }^{36}$ Gruebele et al. ${ }^{35}$ have studied the wavepacket dynamics, product state distributions, and FTS by performing 2-D wave-packet calculations to compare with experiments. The PES we used is that of a damped Morse oscillator with parameters based on spectroscopic data, photofragmentation, I and I* yield, and some $a b$ initio calculations made on analogous systems. The explicit form is given in ref. 35 . 
Wave-packet propagations were done at energies of $-1000,1350$ and $8950 \mathrm{~cm}^{-1}$ with respect to dissociation to $\mathrm{Hg}+\mathrm{I}+\mathrm{I}$ atoms on two surfaces, labelled $(a)$ and $(b)$ in Fig. 11. Fig. 16(A)-(C) show some examples of the dynamics at different excess energies. Fig. 16(A) corresponds to an energy of $-1000 \mathrm{~cm}^{-1}$ with respect to the full $(\mathrm{Hg}+2 \mathrm{I})$ dissociation limit on surface $(b)$. The narrow-channel surface confines the dissociating wave packet, which essentially flows along equipotential contours. Fig. 16(B) corresponds to an energy of $1350 \mathrm{~cm}^{-1}$ on surface $(a)$, and represents the simplest picture of $\mathrm{I}^{*}$ channel dissociation. Here there is considerably more motion in the symmetric coordinate, until the expanding wave packet diffuses back into the walls of the $\mathrm{HgI}$ potential well at $t>300 \mathrm{fs}$. At $800 \mathrm{fs}$, well developed 'clouds' of $\mathrm{Hg}+\mathrm{I}^{*}, \mathrm{I}^{*}+\mathrm{HgI}$ and $I^{*}+\mathrm{Hg}+\mathrm{I}$ have formed. The early-time symmetric stretching behaviour becomes even more dramatic in the case of $c a .8950 \mathrm{~cm}^{-1}$ excitation energy on surface $(b)$, corresponding to the simplest picture of I channel dissociation. The wave packet proceeds along the symmetric coordinate before beginning to separate into three sections. Clearly, the most important motions during the initial phase of the dissociation reaction are not required to be along the natural reaction coordinate (path of steepest descent leading to products) in the present case. While at low energies the steepest descent path is good for parametrization of the reaction, at higher energies motion proceeds along it only at very early times (symmetric coordinate) and very long times (along $r_{1}$ ).

In the asymptotic limit one can obtain approximate vibrational product distributions by taking sections of the wave packet perpendicular to the translational coordinate $r_{1}$, projecting onto the $\mathrm{HgI}\left(\mathrm{X}^{2} \Sigma^{+}\right)$bound eigenstates, and summing the weighted distribution of all sections at different $r_{1}$ to obtain a translationally averaged vibrational distribution (with all coherent effects averaged out). Generally, the average vibrational quantum number decreases with increasing $r_{1}$ of the sections, as shown in Fig. 17, and discussed in more detail in ref. 35.

Vibrational distributions for potentials $(a)$ and $(b)$ at the three energies studied were obtained using these calculations. The wider-channel PES generally causes a marked increase in the vibrational excitation. This is to be expected, since it results in wider ranging initial motion along the symmetric coordinate, which is partially transferred into a larger vibrational amplitude. On both surfaces, as the excess energy is increased, the vibrational distribution broadens considerably and moves to higher average $v^{\prime \prime}$. In addition to the calculations of product-state distributions (Fig. 10 of ref. 35), several quantities, such as emission spectra (Fig. 11 of ref. 35 ) of the probed $\mathrm{HgI}$ and oscillatory effects in the FTS transients (Fig. 18 here) due to wave packet motion, were obtained.

The simplest parameter for comparison with experiment is the reaction time. A long-lived transition state would be reflected in a delay of the experimental transient rise time. In our calculations, the I-channel wave packets reach the asymptotic $(>4.5 \AA)$ region in $c a .200 \mathrm{fs}$, while the $\mathrm{I}^{*}$-channel packet requires $c a .350 \mathrm{fs}$, comparing well with the experimental times for a prompt reaction.

Fig. 18 shows FTS transients for the $I$ and $I^{*}$ channels on surface $(b)$, calculated for a probe wavelength of $390 \mathrm{~nm}$. The periods for both reaction paths correspond closely to the experimental observations. Two features deserve special mention. Because the calculated transients correspond to detection of all the LIF signal (rather than $\lambda_{\text {det }} \pm 5 \mathrm{~nm}$ as in the experiments), and are not convoluted with a 'monochromator response function', they show more structure than experimentally observed. It is also notable that even the fairly broad vibrational distribution at $8950 \mathrm{~cm}^{-1}$ on surface $(b)$ yields sharp, well defined FTS maxima with a 1 ps period. The width of the distribution does lead to some irregularities in the period at long times; indeed, some weak features have been observed ${ }^{28}$ in the experimental transients (Fig. 12) which may correspond to this behaviour.

Finally, the Fourier-transform spectra of the experimental transients reflect the expected time-integrated spectra. In the transition state, the spectra would be $c a$. 
(A)

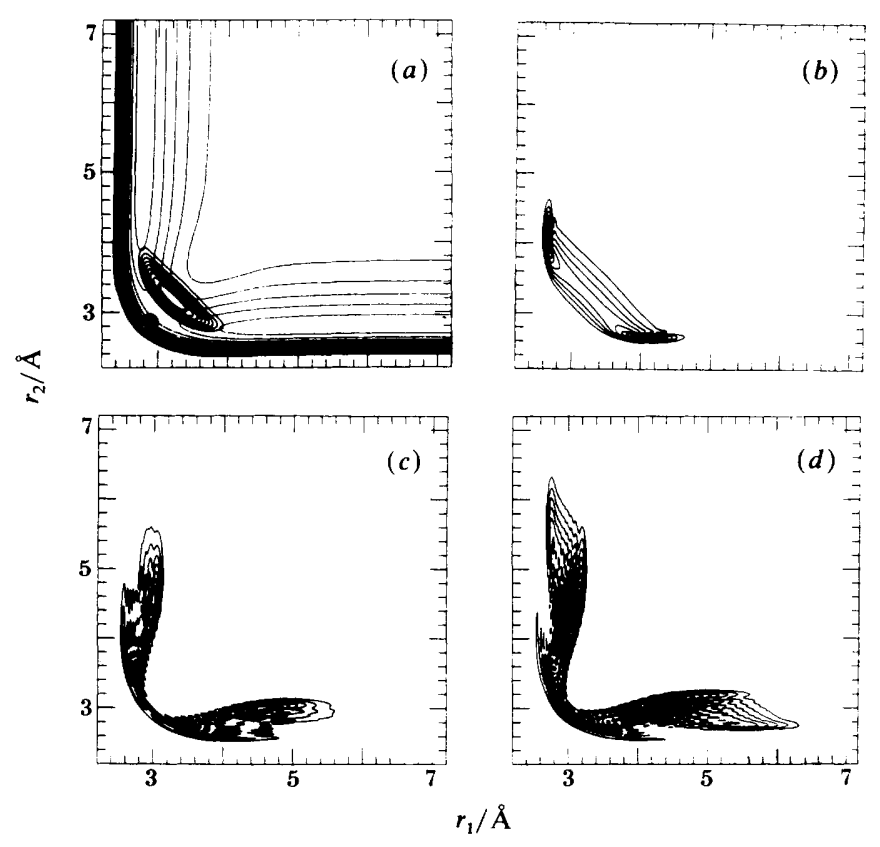

(B)

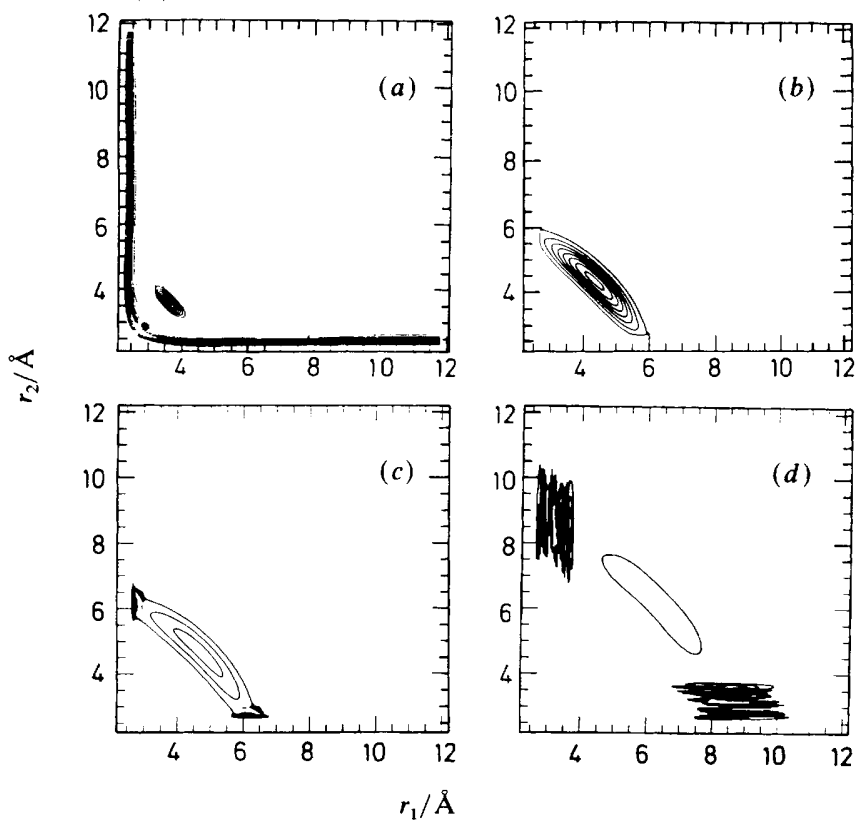

Fig. 16 (A) Snapshots of the wave packet on surface $(b)$ at different times; $(a)=155,(b)=233$, $(c)=389$ and $(d)=467 \mathrm{fs}$. The total energy is $-1000 \mathrm{~cm}^{-1}$ with respect to total dissociation (Fig. 9). (B) The dynamics on surface $(a)$ at $1350 \mathrm{~cm}^{-1}$ with respect to total dissociation, and times $(a) 160,(b) 320,(c) 400 \mathrm{fs}$ and $(d)=800 \mathrm{fs}$. (C) The dynamics on surface $(b)$ at $8950 \mathrm{~cm}^{-1}$ with respect to total dissociation, and times $(a) 58,(b) 233,(c) 400$ and $(d)=600 \mathrm{fs}$ 
(C)

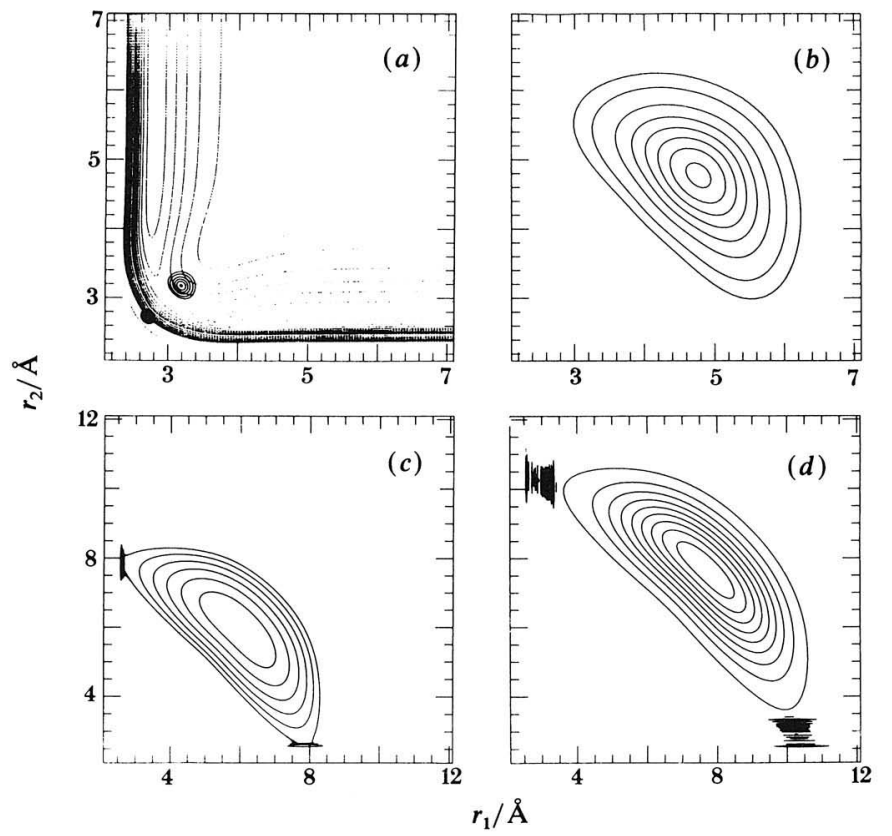

Fig. 16 (continued)

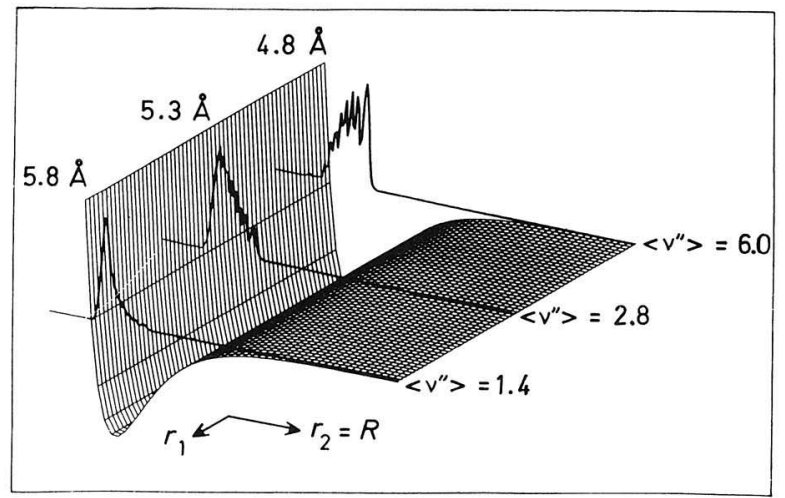

Fig. 17 Slices at three distances along the reaction coordinate $r_{1}$ through the asymptotic region of the packet in Fig. 16(A) $(d)$ at $467 \mathrm{fs}$

$100 \mathrm{~cm}^{-1}$ broad, aside from any resonance effect. On the other hand, as the complex evolves to $\mathrm{HgI}$, the Fourier transform indicates a sharp resonance $\left(\right.$ at $\left.111 \mathrm{~cm}^{-1}\right)$ on a broad background. In the ion photodetachment work, Neumark ${ }^{37}$ has shown characteristics of broad and sharp spectra, and related them to the ultraf ast dynamics by wave-packet simulations. The 3-D quantum-scattering calculations by $S c h a t z^{38}$ elucidate these points.

\subsection{Surface Crossings and Initial Alignment}

The experiments of Hofmann and Leene ${ }^{39}$ have determined the cross-section for the I and $I^{*}$ channels. These experiments can be discussed in terms of excitation to the $I^{*}$ channel surface followed by partial Landau-Zener crossing at a conical intersection 


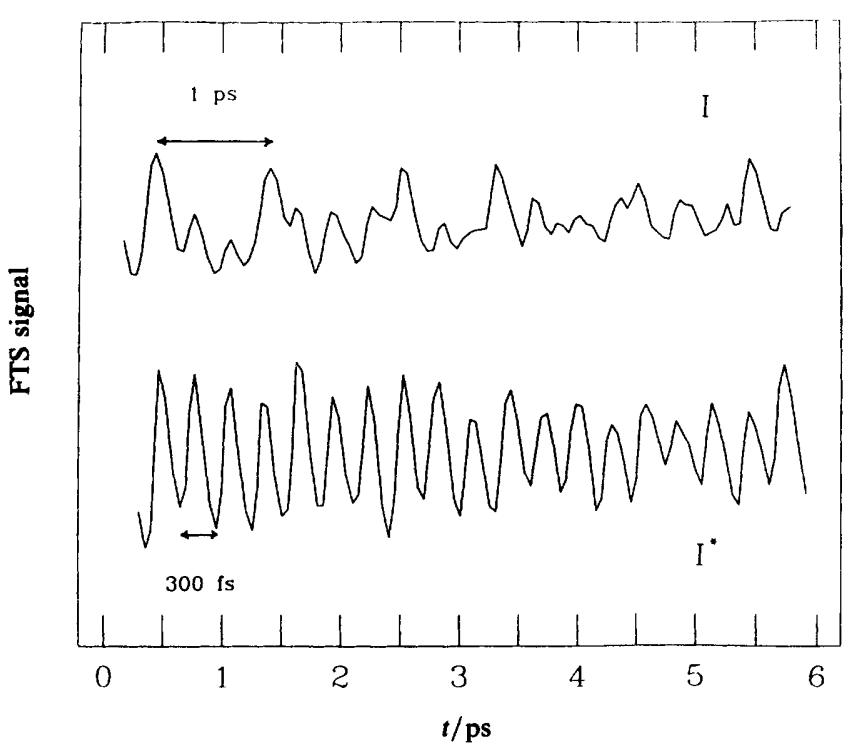

Fig. 18 FTS transients obtained theoretically from the wavepacket propagation on surface (b) at the corresponding total energies (see text)

with the I surface, or in terms of separate excitation to the $I$ and $I^{*}$ surfaces. The $I^{*}$ cross-section reaches a peak at $c a .4300 \mathrm{~cm}^{-1}$ higher photolysis energy than the I crosssection. Alignment experiments in the TS region help establish the nature of crossing (or lack of) between the PESs.

In Fig. 14 the FTS display a strong polarization effect in the first $400 \mathrm{fs}$. If the two PE surfaces of $I$ and $I^{*}$ channels are non-interacting, then an extinction of the polarization is expected. This is because the $I^{*}$ asymptote correlates with a parallel transition, while the I asymptote with a perpendicular transition. ${ }^{40}$ On the other hand, if there is a Landau-Zener interaction, then the states are mixed and the polarization anisotropy of the two channels becomes less pronounced, reaching a limiting value when the mixing is very strong. Our results therefore indicate that the two PE surfaces do not cross at the probe region. There may be a crossing at shorter $R$, but $R$ should be $<3.5 \AA$ to insure that the surfaces are free of interaction at the probing region. These results are consistent with the fact that the second peak (at later time) is the one to achieve enhancement in the $(\|\|$,$) polarization experiment. The behaviour at different detection$ wavelengths (Fig. 14) requires knowledge of $V_{2}$ (with also possible crossing), and the 2-D calculation of the absorption in the TS region. These experiments, when completed at different energies and different $\lambda_{2}$, should provide the nature of the coupling between $P E$ surfaces in the short- $R$ region. It should be noted that on this timescale of the TS, the rotation of the complex is negligible, as evidenced by the measured long coherence time, $1 \mathrm{ps}$, discussed before.

\subsection{Summary and Future Studies}

As discussed above, the uniqueness of this reaction is in showing characteristics of TS dynamics, from a saddle-point region to the asymptotic region (nascent fragment). The early-time transients arise from wave packets moving along the symmetric stretch coordinate (towards total dissociation). At longer times, diffusion into the potential wells sets in, and the vibrational motion of the wave packets gives rise to oscillations 
of the FTS signal. Classical and quantum calculations produce results that are close to experimental observations. These observations include: vibrational oscillations as the complex gives birth to free fragments in different $v$ " states; TS decay, and motion along the symmetric coordinate; the decay (and buildup) of polarization anisotropy, in real time, as bending motion converges to rotations; and the initial alignment $(t<400 \mathrm{fs})$, which reflects the symmetry of and coupling between the PESs.

However, the case of this class of reactions is clearly still far from closed. Experiments at different total energies and different $\lambda_{2}$ would probe the PES at new regions, hopefully for complete characterization, as in the case of alkali halides. The study should also allow us to probe the validity of adiabatic correlation between TS vibrational excitation and coherent formation of vibrational excitation in the product. Characterization of the resonance in the symmetric stretch at different $E$ would be of great interest. Careful alignment studies at several energies should pinpoint the location of surface crossings and the role of the bending vibration in the reaction, starting at different points within the TS region. Product-state distributions will yield valuable information and allow us to compare the coherent dynamical $v^{\prime \prime}$ distribution at early times with the distribution for $t \rightarrow \infty$.

In parallel, $a b$ initio surfaces would be very desirable as a basis for further quantum and classical dynamics studies and more quantitative comparisons between theory and experiments. In particular, three-dimensional calculations, including the bending motion, will then become useful in comparing with the longer-time behaviour of the FTS signal.

Recently, two types of studies have been initiated. On the experimental side, Janssen et $a .^{41}$ have studied the same system (IHgI), but at $\lambda_{1}=335$ and $275 \mathrm{~nm}$. Both the TS and the fragments were detected. The observed oscillations at these energies show some interesting new features, and when these experiments are completed we may learn about the question of adiabatic correlation. Theoreticallyt there is hope for obtaining the calculation of the PES. When available for the IHgI system, we will study the wavepacket dynamics to compare with experiments. Other related systems in the ABA family of reactions are also under experimental investigation.

\section{How Can a Broad-energy Pulse Probe a Sharp Resonance?}

With femtosecond pulses, one immediately thinks of the time resolution dictated by the ultrashort duration. However, according to the uncertainty principle, the pulse will have a broad-energy spectrum, and one might think that if the energy width is much broader than vibrational energy spacings of a resonance (e.g. the symmetric-stretch direction of the PES in section 3), then all the spectroscopic information will be lost. This is not true, provided one can exploit the coherence in the preparation of the complex, as has been observed in the context of vibrational and rotational dynamics of isolated molecules. ${ }^{2}$

Consider a one-dimensional system that can be treated rigorously by classical and quantum mechanics. If a femtosecond laser pulse excites a diatomic molecule, such as $I_{2}$, to the bound region of an excited electronic state, a superposition of vibrational states is formed and then propagates back and forth in the bound well as a wave packet. Probing with a second laser pulse at successive time delays $\Delta t$ should reveal the vibrational and rotational motion; this is possible because the bandwidth of the pump laser is larger than the separation of energy levels. Thus the uncertainty principle works

\footnotetext{
† Progress in this direction has been made for systems of interest $\left(\mathrm{CH}_{3} \mathrm{I}^{42 a} \mathrm{ICN},{ }^{42 b} \mathrm{NaI}^{42 c}\right.$ etc. $)$ by Morokuma's group. For $\mathrm{HgI}_{2}$, Rosmus' group in Frankfurt (personal communication) is initiating studies of the PES.
} 

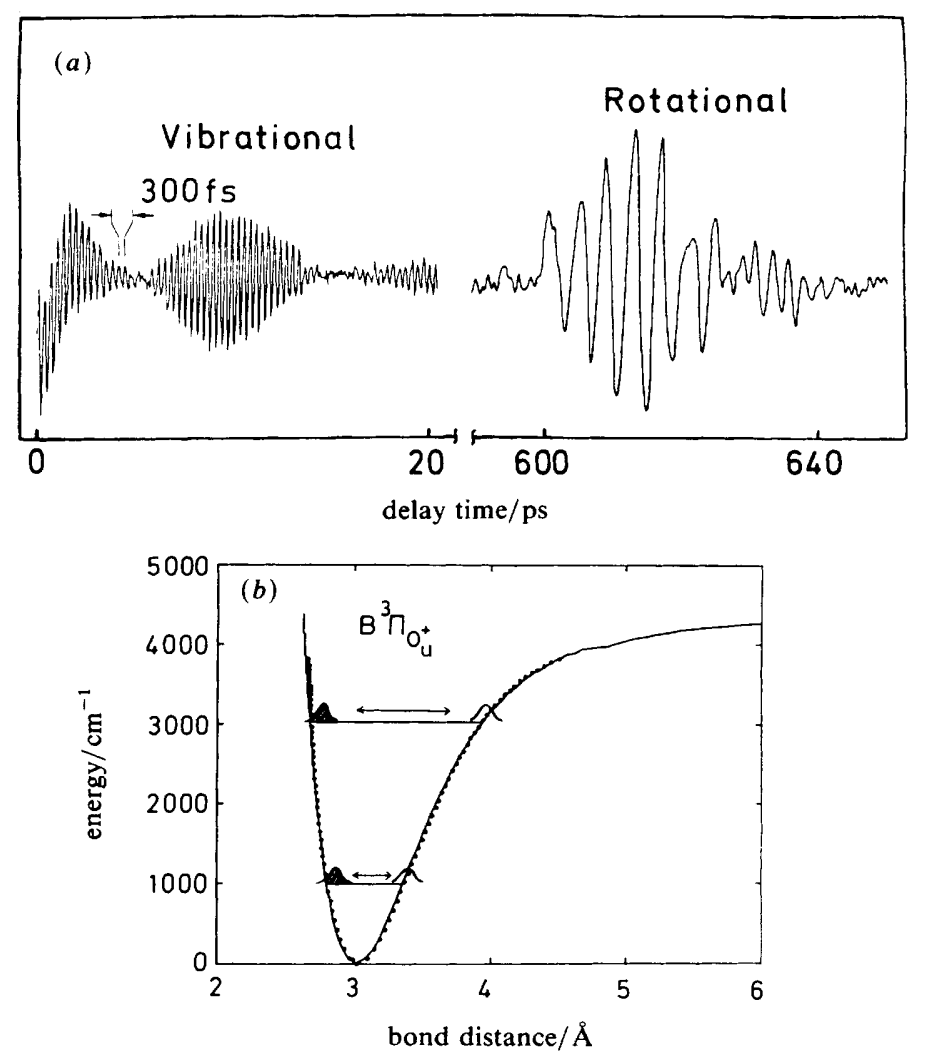

Fig. 19 Experimental FTS results on iodine, showing the transients for both the vibrational and rotational motions $(a)$. In $(b)$ the potential inverted from the FTS data together with that from high-resolution data are compared

in our favour, because the short pulse's finite energy width allows for this coherent superposition! The wave packet moves in and out of resonance with the probe laser absorption window located at a particular internuclear separation. The measured intensity $I(\Delta t, \lambda)$ images the motion and gives the eigenstate frequencies of which the wave packet is composed. Fourier transformation of the intensity $I(\Delta t, \lambda)$ gives spectroscopic data about the bound vibrational states, recovering all the information that one might have thought was lost owing to the broad energy bandwidth of the ultrashort pulses.

If polarized femtosecond pulses are used, the molecules can be aligned, and that alignment can be probed as the molecules rotate in real time, as discussed above for other systems. ${ }^{2}$ The initial alignment decays as the rotational motion of molecules in different angular momentum states dephase, but at longer times the molecules rephase at a time determined precisely by the fundamental rotational period.

Such observations of vibrational and rotational motions have been made for molecular iodine in various excited states by Dantus et al. and Bowman et al. ${ }^{43}$ From these results (Fig. 19) direct inversion to the potential-energy curve governing the I-I vibration has been obtained by Gruebele et al., ${ }^{44}$ Bernstein et al., ${ }^{45}$ and Janssen et al. ${ }^{46}$ The FTS results are in excellent agreement with results from high-resolution techniques. This approach demonstrates two points. First, even though the light pulses are very short and energy resolution is therefore poor, coherent superposition can recover the spectroscopic information, as observed in $\mathrm{NaI}, \mathbf{I}_{2}$ and even in such large molecules as 


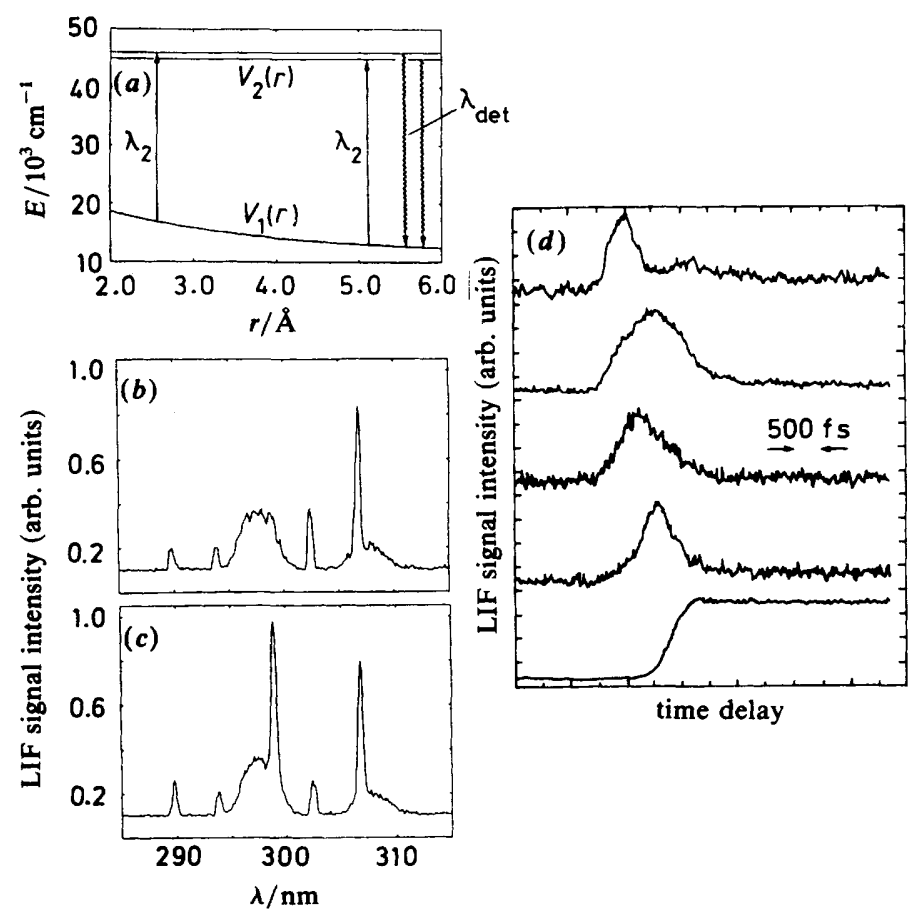

Fig. 20 Recent experimental FTS results on $\mathrm{Bi}_{2}$ with $\lambda_{1}=308 \mathrm{~nm}$ and $\lambda_{2}=298.9 \mathrm{~nm}$. $(a)-(c) \mathrm{A}$ schematic of the potentials $(a)$, and the spectral and temporal evolution of atomic Bi resonance $[(b)-(d)]$; (b) shows the LIF signal taken when the delay time is $t<250 \mathrm{fs}$, while (c) is at $t>1$ ps. Note the absence of one of the sharp lines in $(b)$ and its appearance in $(c)$ as time increases. (The broad bands are the pump and probe spectra on the same scale.) $(d)$ The temporal evolution as $[\mathrm{Bi} \cdots \mathrm{Bi}] \ddagger^{*}$ (top) dissociates to $\mathrm{Bi}+\mathrm{Bi}$ (bottom). Because $\mathrm{Bi}_{2}$ is heavier than, e.g. ICN, the $\mathrm{Bi}$ fragment takes a long time to appear, ca. 1 ps. The results are in agreement with recent quantum and classical calculations, and show the characteristic behaviour for dissociation on a repulsive PES (ref. 2 and 7), but with a long dissociation time [ref. $8(b)]$

anthracene. ${ }^{2}$ Secondly because of the different time scales (and polarization effects) involved in vibrational and rotational motions, the temporal data separate the two, and this separation simplifies the analysis considerably; in these femtosecond experiments, we used only six measurements to obtain the potential shown in Fig. 19. Finally, in simple bound systems, the equivalence of energy- and time-resolved techniques is clear. The situation is different for transition states, however, owing to their ultrashort lifetimes and broad background spectra.

It is therefore evident that resonance effects in the TS dynamics can be detected using ultrashort pulses.

\section{Other Studies and Future Directions}

Since the early development of this field, a number of reactions have been studied. In ref. 2 the progress made was recently reviewed. The concepts and methodology have been illustrated with examples drawn on increasingly complex potential-energy surfaces, from bound diatomics, to 1-D direct reactions, to reactions involving potentials with avoided crossings, to $2-\mathrm{D}$ half-collision reactions and finally to a special class of bimolecular reactions: (i) bound systems: $\mathrm{I}_{2},{ }^{43-45} \mathrm{ICl}^{46}$ (ii) 1-D repulsive systems: $\mathrm{I}_{2},{ }^{43}$ $\mathrm{ICN}^{7}$ and $\mathrm{Bi}_{2}{ }^{8}$ (Fig. 20); (iii) systems with avoided crossing: $\mathrm{NaI}$ and $\mathrm{NaBr} ;{ }^{6,15,17}$ (iv) 
2-D (or more) systems: $\mathrm{HgI}_{2}{ }^{28,35}$ and $\mathrm{CH}_{3} \mathrm{I}^{47,48}$ (v) a special class of bimolecular species: IH $\cdots \mathrm{CO}_{2}{ }^{49}$ The IBM group has provided experimental and theoretical studies of time-resolved absorption, on the sub-picosecond timescale, which illustrate the direct dissociation dynamics in $\mathrm{Bi}_{2}$ and $\mathrm{Tl} \mathrm{I}^{8 a, 50}$ In Freiburg, the femtosecond dynamics of autoionization has been demonstrated on $\mathrm{Na}_{2} \cdot{ }^{51}$ On the theory side, a large number of contributions have been made, and ref. 2 gives a recent review of the work done by many groups.

There are other directions that promise to be important. Experimentally, the use of multiphoton probing or excitation should allow one to reach different PE surfaces (see ref. 48, 51 and 52). Very recently, using molecular beam mass-spectrometry, M. Janssen and $M$. Dantus (this laboratory) have observed the femtosecond predissociation dynamics of molecules in Rydberg states (ca. 8-9 eV energy), using a total of two UV photons and one red. This study ${ }^{48}$ extends FTS to a new direction for probing the dynamics of highly excited molecules, and so far $\mathrm{CH}_{3} \mathrm{I}, \mathrm{CD}_{3} \mathrm{I},\left(\mathrm{CH}_{3}\right)_{3} \mathrm{CI}$ and $\mathrm{I}_{2}$ have been studied in molecular beams. With the use of depletion and stimulated emission techniques, one should be able to reach ground state PESs. Double-resonance methods in the preparation may help in reaching a well defined and different initial state.

To date, LIF, ${ }^{2}$ ionization ${ }^{2}$ and mass-spectrometric ${ }^{2,48,51}$ detection techniques have all been employed. The use of Raman and IR techniques is another advance that would complement these techniques. To obtain direct structural information on the femtosecond timescale, it is proposed to extend FTS by 'replacing' the probe optical pulse with an electron pulse so that femtosecond transition-state diffraction (FTD) can be obtained. The trajectories $R(t)$ will then per se be observed for all atoms. The technique promises to be general for recording elementary and complex reactions and for studying molecular rearrangements. Such an FTD 'machine' is currently under construction at Caltech to cover the PS to FS time domains. Extension of this concept to X-rays may also be possible in the future.

There are a number of systems, small and large, to be studied using these techniques, and in the coming years we anticipate extensions to new areas: reactions on surfaces; large organics, and ionic systems; other van der Waals impacted bimolecular reactions (and even 'honest' bimolecular reactions). The class of bimolecular reactions light-heavy-heavy versus heavy-light-heavy $(\mathrm{HX} \cdot \mathrm{Y}$ and $\mathrm{X} \cdot \mathrm{H} \cdot \mathrm{Y}$, where $\mathrm{X}$ and $\mathrm{Y}$ are atoms and diatoms, e.g. halogens) are of particular interest to us. We hope to be able to observe the oscillatory motion of the activated complex (Fig. 21), similar to the case of alkali halides and $\mathrm{HgI}_{2}$, but now on the ground-state PES of a bimolecular reaction. One significant advance here is the ability to study the bimolecular collision for systems like $\mathrm{HBr} \cdot \mathrm{I}_{2}$, where the light $\mathrm{H}$ atom is $c a .20 \AA$ away from the force field of the reactants within $100 \mathrm{fs}$. These studies will be made using state-of-the-art femtosecond pulses and molecular beams. The same apparatus is currently used to resolve the femtosecond dynamics of the $\mathrm{XH} \cdot \mathrm{CO}_{2}$ system (see ref. 49 for discussion), which so far has been studied in the ps regime. Studies of the inelastic scattering would also be of great interest.

Control of wave-packet motion may be at the moment an academic curiosity; however, it is of great interest. In the iodine experiments by Gerdy et al. ${ }^{53}$ the control of the packet in the preparation process was made using two pulses of well defined relative phases. This sequence of pulses changes the amplitudes and hence the apparent simple oscillatory behaviour observed earlier using conventional FTS on $\mathrm{I}_{2}{ }^{43}$ Wavepacket theory ${ }^{54}$ is in agreement with experiments. Femtosecond phase controlled pulses by Scherer et al. ${ }^{55}$ have been demonstrated on $\mathrm{I}_{2}$, also in the gas phase, and wave-packet theory is in accord with experiments. Weiner et al. ${ }^{56}$ have reported on phase-controlled impulses in crystals, and Mukamel ${ }^{56}$ has discussed the theory under these conditions. The iodine experiments indicate that both the amplitude and phase of the motion are controllable. In this area, theory ${ }^{57}$ has been very enlightening and different proposals have been introduced by Brumer and Shapiro, Tannor and Rice, Rabitz, Manz and others. 


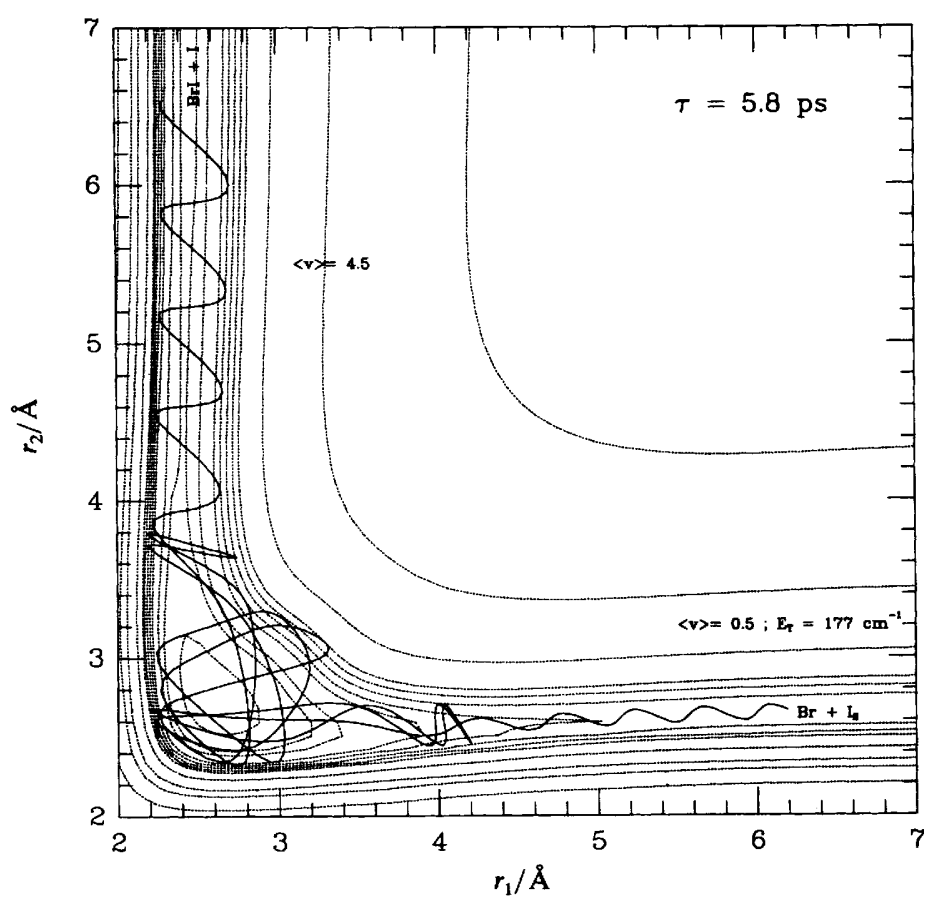

Fig. 21 Femtosecond dynamics of a bimolecular reaction: $\mathrm{Br}+\mathrm{I}_{2} \rightarrow \mathrm{IBr}+\mathrm{I}$. The calculations were made by $M$. Gruebele (this laboratory) and they demonstrate the oscillatory motion of the complex $[\mathrm{Br} \cdots \mathrm{I} \cdots \mathrm{I}] . \neq$ In real time, analogy with the results of Fig. 2 should be made. The lifetime of the complex resonance in this case is $c a .6 \mathrm{ps}$ at the translational energy of interest for this potential

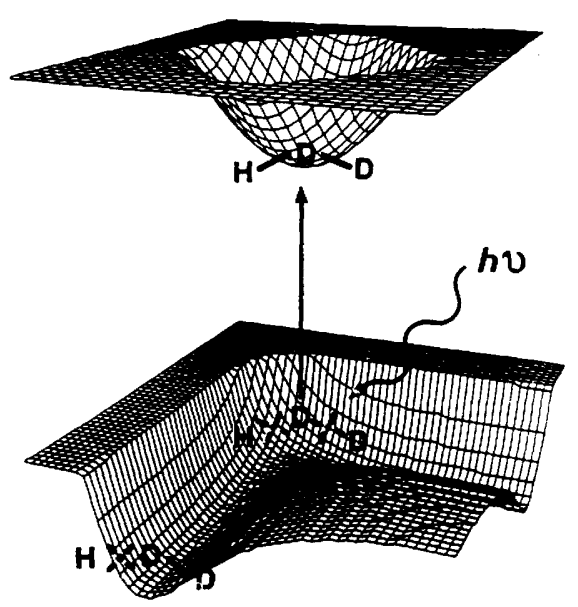

Fig. 22 The idea of TS spectroscopy of $H / D_{2}$ system, courtesy of John Polanyi's group (ref. 70); the 'inverse case' of IHgI (see text) 
Finally, the TS spectroscopy of the $\mathrm{H}+\mathrm{D}_{2}$ system was pioneered by Polanyi's group. ${ }^{58}$ The experiments, although shown to be difficult, ${ }^{58}$ are important for a number of theoretical and experimental reasons. We hope to be able to carry out such experiments $\left(\mathrm{F}+\mathrm{H}_{2}\right.$ and $\mathrm{H}+\mathrm{D}_{2}$ ) now with FTS (Fig. 22) when the required sensitivity and temporal resolution are attained. The experiments will be similar to the $\mathrm{HI}: \mathrm{CO}_{2}$ study, ${ }^{49}$ but with ca. 6 fs time resolution. We will compare the results with those made by photodetachment in real-time by using FTS and invoking Neumark's idea of going from the anion to the TS.

Much remains to be studied in femtosecond transition-state dynamics and the femtochemistry of reactions. Have the experiments reached the so-called 'chemistry timescale'? In $6 \mathrm{fs}$, the nuclear motions are indeed those which characterize chemical reactions and molecular dynamics. ${ }^{2}$ For reactions, this time corresponds, typically, to a motion of $c a .0 .1 \AA$, and it is shorter than any TS lifetime (deduced by classical and quantum calculations). ${ }^{2}$ The $6 \mathrm{fs}$ duration represents the state of the art in laser pulse generation achieved by Shank and colleagues. ${ }^{59}$ The energy uncertainty $\Delta E$, although not a problem for the preparation even in bound systems (see section 4), should be compared with bond energies; $\Delta E$ is $0.7 \mathrm{kcal} \mathrm{mol}^{-1}$ for a $60 \mathrm{fs}$ pulse and $7 \mathrm{kcal} \mathrm{mol}^{-1}$ for a $6 \mathrm{fs}$ pulse. $\dagger$ So does it help to shorten the pulse further? One may say not, only because the energy uncertainty of sub-femtosecond pulses (attosecond regime) is very large $\left(100\right.$ as $\left.\equiv 420 \mathrm{kcal} \mathrm{mol}^{-1}\right)$ compared to bond energies, as pointed out by Porter ${ }^{60}{ }_{\ddagger} \ddagger$ As illustrated here (section 4), despite the broadening, the pulse can still be used for coherent preparation, and this 'avoids' the issue of the uncertainty principle.

While sub-femtosecond pulses' resolution may be considered outside the 'limit of chemistry', ${ }^{61}$ they may prove useful for electron motion and valency. For example, based on Pauling's ${ }^{4}$ simple description of the bonding in $\mathrm{H}_{2}^{+}$, the electron will hop between the two nuclei in a time of $2 \mathrm{fs}$ (about an order of magnitude longer than the orbital motion about the nucleus of the electron in the hydrogen atom) because of the $50 \mathrm{kcal} \mathrm{mol}^{-1}$ resonance energy. The localization length of the initial packet must be on the atomic or molecular scale, still orders of magnitude larger than the scale of femtophysics $(1 \mathrm{fm}) .^{62}$ Perhaps attosecond pulses would allow us to see such the process of electron valency (in $\mathrm{H}_{2}^{+}$, benzene structures etc.) in real time, just as femtosecond pulses make it possible to expose ${ }^{63}$ the (nuclear) dynamics of the chemical bond.

This research was supported by the Air Force Office of Scientific Research and by the National Science Foundation. It is with pleasure that $I$ thank members of my group for all their help and discussions during the writing of the manuscript. I would particularly like to thank Gareth Roberts for his help and thorough reading of the manuscript. I also thank Peijun Cong, Martin Gruebele, Bob Bowman and Jim Gerdy for all their efforts. In discussing the current work on bimolecular reactions with Prof. John Polanyi during a recent visit to Caltech, he suggested the extension of the studies to include inelastic scattering of the products; we hope to be able to study both the reactive and inelastic collisions. Finally, I thank Professor John Simons for his patience and kindness; E-mail did help in getting the manuscript over the barrier (transition-state) to overseas!

\section{Dedication}

Richard B. Bernstein, a dear friend and colleague, was scheduled to give the Spiers Memorial Lecture of this Discussion. Dick was planning to talk about the dynamics of

$\dagger 1 \mathrm{cal}=4.184 \mathrm{~J}$

$\ddagger$ Ref. 60 indicates that a $1 \mathrm{fs}$ pulse has a broadening of $400 \mathrm{k} \mathrm{J} \mathrm{mol}^{-1}\left(=95.6 \mathrm{kcal} \mathrm{mol}^{-1}\right)$, simply by using $\Delta t \Delta \nu=1$. In our calculation, we use experimental transform-limited pulses, and for gaussian pulses this relationship is actually $\Delta t \Delta \nu=0.441$. Thus, $1 \mathrm{fs} \equiv 42 \mathrm{kcal} \mathrm{mol}^{-1}$ or $176 \mathrm{~kJ} \mathrm{~mol}^{-1}$. For sech - pulses, $\Delta t \Delta \nu=$ 0.315 . 
bimolecular reactions in real time, mentioned in the text, for work completed during two sabbatical leaves at Caltech. This article is dedicated to the memory of Dick; his friendship, excitement about femtochemistry, and the joy he brought in discussing science will never be forgotten.

\section{References}

1 H. Eyring, J. Chem. Phys., 1935, 3, 107; M. G. Evans and M. Polanyi, Trans. Faraday Soc., 1935, 31, 875; for recent reviews see D. G. Truhlar, W. L. Hase and J. T. Hynes, J. Phys. Chem., 1983 87, 2664 and K. J. Laidler and M. C. King, J. Phys. Chem., 1983, 87, 2657.

2 For recent reviews see L. R. Khundkar and A. H. Zewail, Ann. Rev. Phys. Chem., 1990, 41, 15; A. H. Zewail and R. B. Bernstein, Chem. Eng. News, 1988 66, 24; A. H. Zewail, Science, 1988 242, 1645.

3 R. S. Berry, in Alkali Halide Vapors, ed. P. Davidovits and D. L. McFadden, Academic Press, New York, 1979 and references therein.

4 L. Pauling, The Nature of the Chemical Bond, Cornell University Press, Ithaca, NY, 3rd edn., 1960.

5 P. W. Atkins, Physical Chemistry, Oxford University Press, Oxford, 4th edn., p. 856.

6 T. S. Rose, M. J. Rosker and A. H. Zewail, J. Chem. Phys., 1988, 88, 6672; M. J. Rosker, T. S. Rose and A. H. Zewail, Chem. Phys. Lett., 1988, 146, 175; T. S. Rose, M. J. Rosker and A. H. Zewail, J. Chem. Phys., 1989, 91, 7415.

7 M. Dantus, M. J. Rosker and A. H. Zewail, J. Chem. Phys., 1987, 87, 2395; M. J. Rosker, M. Dantus and A. H. Zewail, J. Chem. Phys., 1988, 89, 6113; M. Dantus, M. J. Rosker and A. H. Zewail, J. Chem. Phys., 1988, 89, 6128; G. Roberts and A. H. Zewail, J. Phys. Chem., 1991, 95, 7973.

8 (a) J. H. Glownia, J. A. Misewich and P. P. Sorokin, J. Chem. Phys., 1990, 92, 2335; J. A. Misewich, J. H. Glownia, R. E. Walkup and P. P. Sorokin, in Ultrafast Phenomena VII, Springer Series in Chemical Physics, vol. 53, ed., C. B. Harris, E. P. Ippen, G. A. Mourou and A. H. Zewail, Springer-Verlag, New York, 1990, p. 426; (b) R. M. Bowman, J. J. Gerdy, G. Roberts and A. H. Zewail, J. Phys. Chem., 1991, 95, 4635 .

9 R. B. Bernstein and A. H. Zewail, J. Chem. Phys., 1989, 90, 829.

10 M. J. Rosker, M. Dantus and A. H. Zewail, Science, 1988, 241, 1200.

11 R. Bersohn and A. H. Zewail, Ber. Bunsenges. Phys. Chem., 1988, 92, 373.

12 R. D. Bower, P. Chevrier, P. Das, H. J. Foth, J. C. Polanyi, M. G. Prisant and J. P. Visticot, J. Chem. Phys., 1988, 89, 4478.

13 H. Bluhm, J. Lindner and E. Tiemann, J. Chem. Phys., 1990, 93, 4556; S. H. Schaefer, D. Bender and E. Tiemann, Chem. Phys., 1984, 89, 65; Chem. Phys. Lett., 1982, 92, 273.

14 R. Grice and D. R. Herschbach, Mol. Phys., 1974, 27, 159; see also S. A. Adelman and D. R. Herschbach, Mol. Phys., 1977, 33, 793.

15 P. Cong, A. Mokhtari and A. H. Zewail, Chem. Phys. Lett., 1990, 172, 109.

16 J. A. Beswick and J. Jortner, Chem. Phys. Lett., 1990, 168, 246.

17 A. Mokhtari, P. Cong, J. L. Herek and A. H. Zewail, Nature (London), 1990, 348, 225.

18 W. H. Knox, R. S. Knox, J. F. Hoose and R. N. Zare, Opt. Photonics News, 1990, April, 44.

19 S.-Y. Lee, W. T. Pollard and R. A. Mathies, J. Chem. Phys., 1989, 90, 6146.

20 R. A. Marcus, Chem. Phys. Lett., 1988, 152, 8.

21 S. H. Lin and B. Fain, Chem. Phys. Lett., 1989 155, 216; S. H. Lin, B. Fain and C. Y. Yeh, Phys. Rev. A, 1990, 41, 2718; S. H. Lin, B. Fain and N. Hamer, Adv. Chem. Phys., 1990, 79, 133.

22 V. Engel and H. Metiu, Chem. Phys. Lett., 1989 155, 77; J. Chem. Phys., 1989, 90, 6116; J. Chem. Phys., 1989, 91, 1596; V. Engel, M. Metiu, R. Almeida, R. A. Marcus and A. H. Zewail, Chem. Phys. Lett., 1988, 152, 1.

23 S. E. Choi and J. C. Light, J. Chem. Phys., 1989, 90, 2593.

24 S. Chapman and M. S. Child, J. Phys. Chem. 1991, 95, 578.

25 H. Kono and Y. Fujimura, Chem. Phys. Lett., 1991, 184, 497.

26 R. B. Bernstein, in Proc. The Robert A. Welch Conference on Chemical Research, XXXII Valency, Texas, 1988 , p. 221.

27 G. G. Balint-Kurti and M. Shapiro, in Photodissociation and Photoionization, ed. K. P. Lawley, Wiley, New York, 1985, p. 403; K. F. Freed and Y. B. Band, in Excited States, ed. E. C. Lim, Academic Press, New York, 1978, vol. 3, p. 109; K. C. Kulander and E. J. Heller, J. Chem. Phys., 1978, 69, 2439; J. P. Simons and P. W. Tasker, Mol. Phys., 1973, 26, 1267; 1974, 27, 1691; R. T. Pack, J. Chem. Phys., 1976, 65, 4765; R. Schinke, J. Phys. Chem., 1988, 92, 3195 and references therein; R. D. Coalson and M. Karplus, J. Chem. Phys., 1990, 93, 3919; J. Jiang and J. S. Hutchinson, J. Chem. Phys., 1987, 87, 6973; R. Marquardt and M. Quack, J. Chem. Phys., 1989, 90, 6320.

28 R. M. Bowman, M. Dantus and A. H. Zewail, Chem. Phys. Lett., 1989, 156, 131; M. Dantus, R. M. Bowman, M. Gruebele and A. H. Zewail, J. Chem. Phys. 1989, 91, 7437. 
29 N.-H. Cheung and T. A. Cool, J. Quant. Spectrosc. Radiat. Transfer, 1979, 21, 397; J. A. McGarvey Jr., N.-H. Cheung, A. C. Erlandson and T. A. Cool, J. Chem. Phys., 1981, 74, 5133.

30 C. H. Greene and R. N. Zare, Ann. Rev. Phys. Chem., 1982, 33, 119; G. E. Hall and P. Houston, Ann. Rev. Phys. Chem., 1989, 40, 375; R. B. Bernstein, D. R. Herschbach and R. D. Levine, J. Phys. Chem., 1987, 91, 5365; J. P. Simons, J. Phys. Chem., 1987, 91, 5378.

31 P. M. Felker and A. H. Zewail, J. Chem. Phys., 1987, 80, 2460.

32 A. H. Zewail, J. Chem. Soc., Faraday Trans. 2, 1989, 85, 1221; M. Dantus, R. M. Bowman, J. S. Baskin and A. H. Zewail, Chem. Phys. Lett., 1989, 159, 406.

33 D. R. Herschbach, Faraday Discuss. Chem. Soc., 1973, 55, 233; D. R. Herschbach, Angew. Chem., Int. Ed. Engl., 1987, 26, 1221.

34 J. C. Polanyi, Science, 1987, 236, 680.

35 M. Gruebele, G. Roberts and A. H. Zewail, Philos. Trans. R. Soc. London, Ser. A, 1990, $332,223$.

36 E. J. Heller, Acc. Chem. Res., 1981, 14, 368; S. O. Williams and D. G. Imre, J. Phys. Chem., 1988,92, 6636; 6648; V. Engel and H. Metiu, J. Chem. Phys., 1990, 92, 2317; H. Metiu and V. Engel, J. Chem. Phys., 1990, 93, 5693.

37 A. Weaver and D. M. Neumark, Faraday Discuss. Chem. Soc., 1991, 91, 5 and references therein; see also D. M. Neumark, Adv. Chem. Phys., in the press; S. E. Bradforth, A. Weaver, D. W. Arnold, R. B. Metz and D. M. Neumark, J. Chem. Phys., 92, 1990, 7205.

38 G. C. Schatz, J. Phys. Chem., 1990, 94, 6157.

39 H. Hofmann and S. R. Leone, J. Chem. Phys., 1978, 69, 3819.

40 See e.g., M. Kawasaki, S. J. Lee and R. Bersohn, J. Chem. Phys., 1979, 71, 1235, and references therein.

41 M. H. M. Janssen and M. Dantus, unpublished work from this laboratory.

42 (a) S. Yabushita and K. Morokuma, Chem. Phys. Lett., 1988, 153, 517; (b) Chem. Phys. Lett., 1990 175, 518; (c) Faraday Discuss. Chem. Soc., 1991, 91, 47.

43 R. M. Bowman, M. Dantus and A. H. Zewail, Chem. Phys. Lett., 1989, 161, 297; M. Dantus, R. M. Bowman and A. H. Zewail, Nature (London), 1990, 343, 737.

44 M. Gruebele, G. Roberts, M. Dantus, R. M. Bowman and A. H. Zewail, Chem. Phys. Lett., 1990, 166, 459.

45 R. B. Bernstein and A. H. Zewail, Chem. Phys. Lett., 1990, 170, 321.

46 M. H. M. Janssen, R. M. Bowman and A. H. Zewail, Chem. Phys. Lett., 1990, 172, 99; M. H. M. Janssen and A. H. Zewail, to be published.

47 L. R. Khundkar and A. H. Zewail, Chem. Phys. Lett., 1987, 142, 426.

48 M. Dantus, M. H. M. Janssen and A. H. Zewail, Chem. Phys. Lett., 1991, 181, 281.

49 N. F. Scherer, L. R. Khundkar, R. B. Bernstein and A. H. Zewail, J. Chem. Phys., 1987, 87, 1451; N. F. Scherer, C. Sipes, R. B. Bernstein and A. H. Zewail, J. Chem. Phys., 1990, 92, 5239.

50 R. E. Walkup, J. A. Misewich, J. H. Glownia and P. P. Sorokin, Phys. Rev. Lett., 1990, 65, 2366 and references therein.

51 T. Baumert, B. Bühler, R. Thalweiser and G. Gerber, Phys. Rev. Lett., 1990, 64, 733; G. Gerber, Faraday Discuss. Chem. Soc., 1991, 91, 358.

52 R. M. Bowman, M. Dantus and A. H. Zewail, Chem. Phys. Lett., 1990, 174, 546.

53 J. J. Gerdy, M. Dantus, R. M. Bowman and A. H. Zewail, Chem. Phys. Lett., 1990, 171, 1.

54 B. Hartke, Chem. Phys. Lett., 1990, 175, 322.

55 N. F. Scherer, A. J. Ruggiero, M. Du, and G. R. Fleming, J. Chem. Phys., 1990, 93, 856; N. Scherer, R. Carlson, A. Matro, M. Du, A. Ruggiero, V. Romero-Rochin, J. Cina, G. Fleming and S. Rice, to be published.

56 A. M. Weiner, D. E. Leaird, G. P. Wiederrecht and K. A. Nelson, Science, 1990, 247, 1317; S. Mukamel and Y.-J. Yan, J. Phys. Chem., 1991, 95, 1015.

57 P. Brumer and M. Shapiro, Acc. Chem. Res., 1989, 22, 407; D. J. Tannor and S. A. Rice, J. Chem. Phys., 1985, 83, 5013; H. Rabitz, in Atomic and Molecular Processes with Short Intense Laser Pulses, ed. A. Bandrank, Plenum Press, New York, 1988; B. Hartke, J. Manz and J. Mathis, Chem. Phys. Lett., 1989, 139, 123; B. Hartke, E. Kolba, J. Manz and H. H. R. Schor, Ber. Bunsenges. Phys. Chem., 1990, 94, 1312 and references therein.

58 B. A. Collins, J. C. Polanyi, M. A. Smith, A. Stolow and A. W. Tarr, Phys. Rev. Lett., 1987, 59, 2551; $1988,60,383 ; 1989,63,2160 ; 1990,64,238$; see also H. R. Mayne, R. A. Poirier and J. C. Polanyi, J. Chem. Phys., 1984, 80, 4025.

59 C. V. Shank, Science, 1986, 233, 1276 and references therein.

60 G. Porter, in Picosecond Chemistry and Biology, ed. T. A. M. Doust and M. A. West, Science Reviews, Northwood, UK.

61 P. C. Jordan, Chemical Kinetics and Transport, Plenum Press, New York, 1979, p. 82.

62 M. G. Bowler, Femtophysics, Pergamon Press, Oxford, 1990.

63 I. W. M. Smith, Nature (London), 1990, 343, 691.

64 G. A. L. Delvigne and J. Los, Physica, 1973, 67, 166; G. A. L. Delvigne and J. Los, Physica, 1972, 59, 61; A. M. C. Moutinho, J. A. Aten and J. Los, Physica, 1971, 53, 471.

65 N. J. A. van Veen, M. S. de Vries, J. D. Sokol, T. Baller and A. E. de Vries, Chem. Phys., 1981, 56,81 . 
66 J. Wang, A. J. Blake, D. G. McCoy and L. Torop, Chem. Phys. Lett., 1990, 175, 225.

67 M. B. Faist and R. D. Levine, J. Chem. Phys., 1976, 64, 2953.

68 Work to be published from this laboratory.

69 R. B. Bernstein, Chemical Dynamics via Molecular Beam and Laser Techniques Oxford University Press, Oxford, 1982.

70 The graphics are taken from P. J. Andrews, Mosaic, 1989, 20 (2), 36.

Paper 1/00399B; Received 29th January, 1991 